\title{
Generation of anisotropic gold and gold- palladium bimetallic nanoparticles on functionalized surfaces
}

\section{Veronica L. Gerios}




\section{Organization}

\section{In situ Generated Metal Nanoparticles}

$\odot$ AuPd Bimetallic Nanoparticles on Surface (catalyst)

$\odot$ Au Nanorods on Surface (catalyst) 


\section{Bimetallic NPs Overview}

Boron-doped

Silicon or

Indium Tin

Oxide Glass

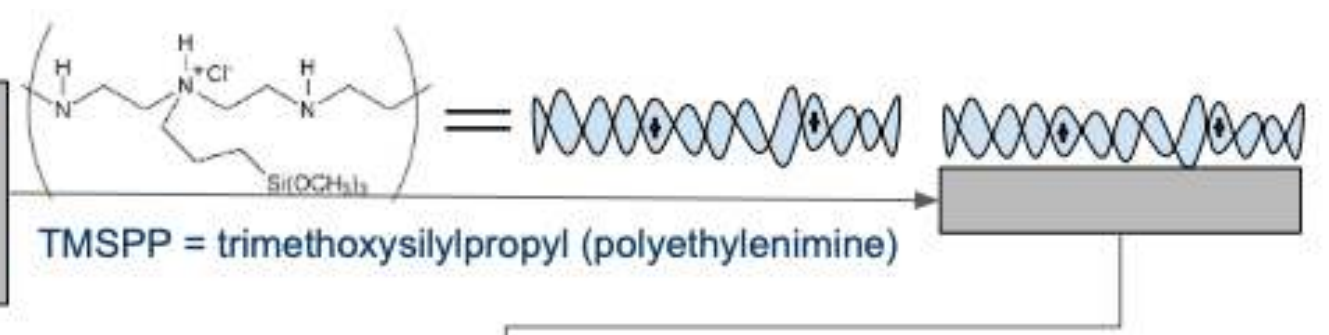

Allow ions to adsorb on surface for 8 hours in solutions with volumetric ratios of $1 \times 10^{-2} \mathrm{M} \mathrm{K}_{2} \mathrm{PdCl}_{4} \& 1 \times 10^{-2} \mathrm{M} \mathrm{HAuCl}_{4}$

(1.) (1.) (2.) (2.) 20 2. 0000000000000000000000000000000000000000000000000000000000 $1: 0$ $1: 0.5$
$0.5: 1$ $0: 1$

Reduce for 2 hours in $0.01 \%(w / v)$ sodium borohydride

$\bigcap_{\infty \times 0 \times \infty \times \infty}$

$1: 0$

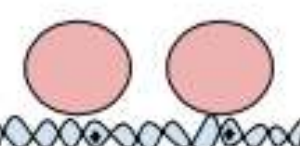

$1: 0.5$

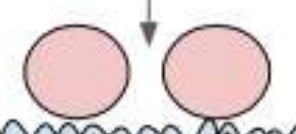

$1: 1$

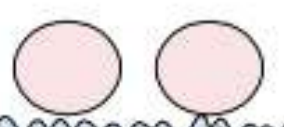

$0.5: 1$

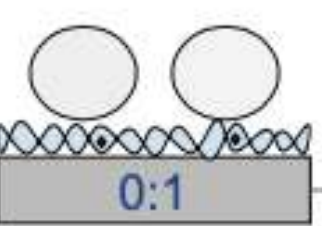

$\rightarrow$ Impedance

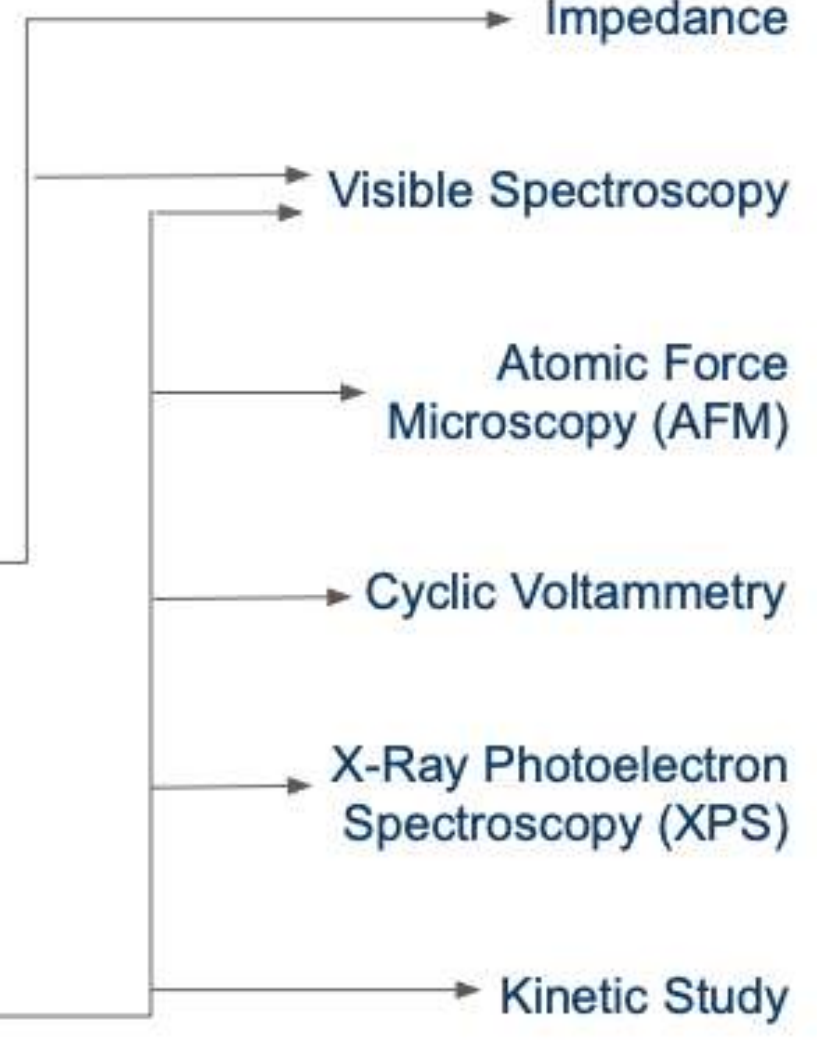




\section{Background}

Hong, Y. ACS Sustainable Chem. Eng. 2014, 2, 1752-1759.

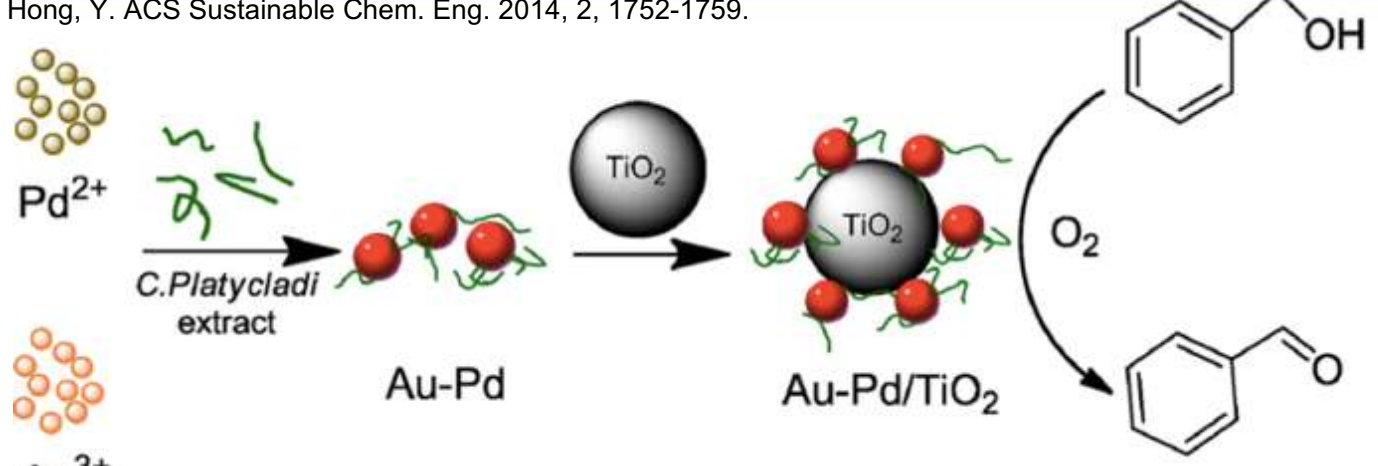
$\mathrm{Au}^{3+}$
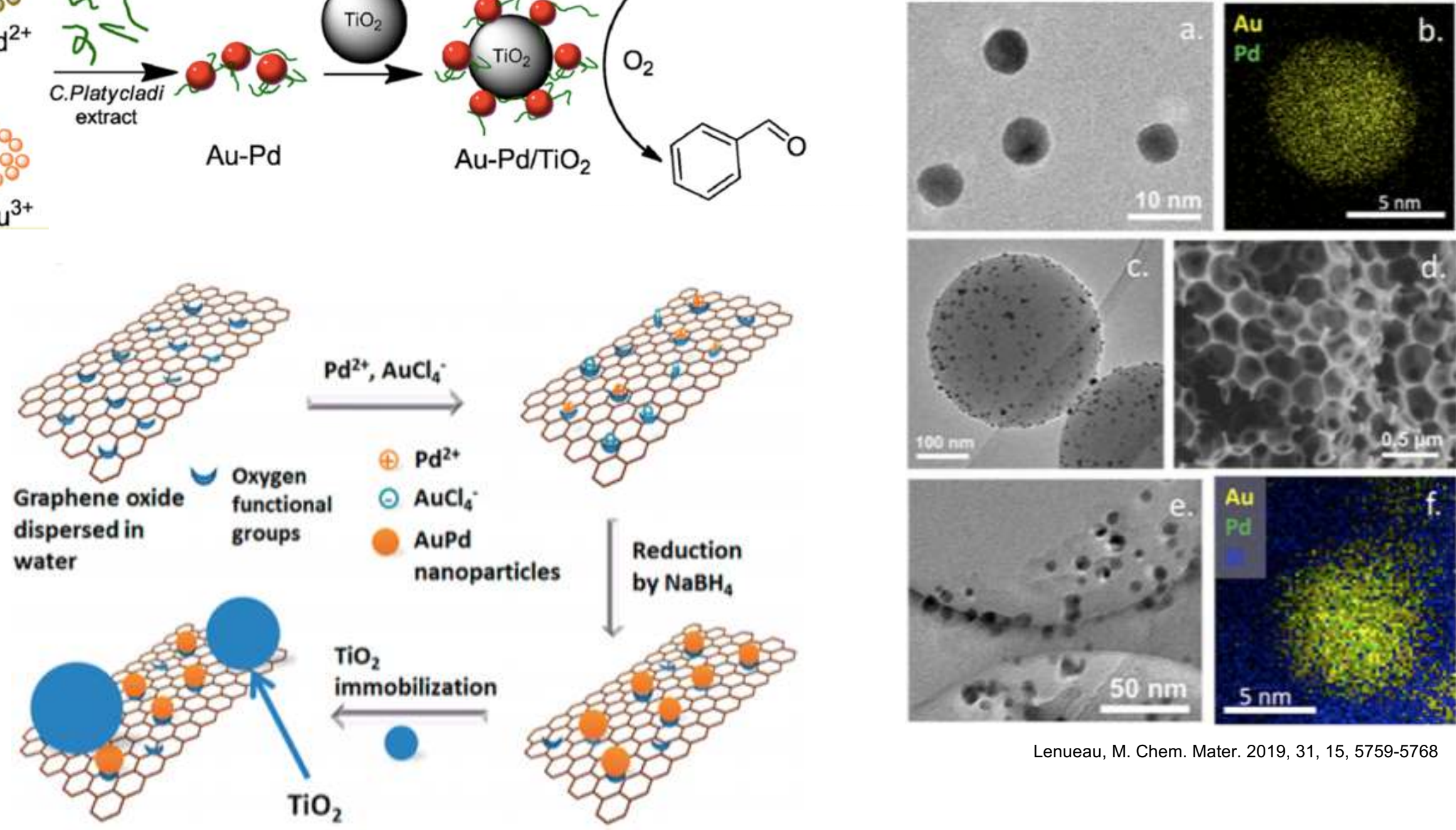

Lenueau, M. Chem. Mater. 2019, 31, 15, 5759-5768

Wang, J. ACS Catal. 2015, 5, 3575-3587 


\section{In-situ Generation of NPs on Surfaces}

Boron-doped

Silicon or

Indium Tin

Oxide Glass

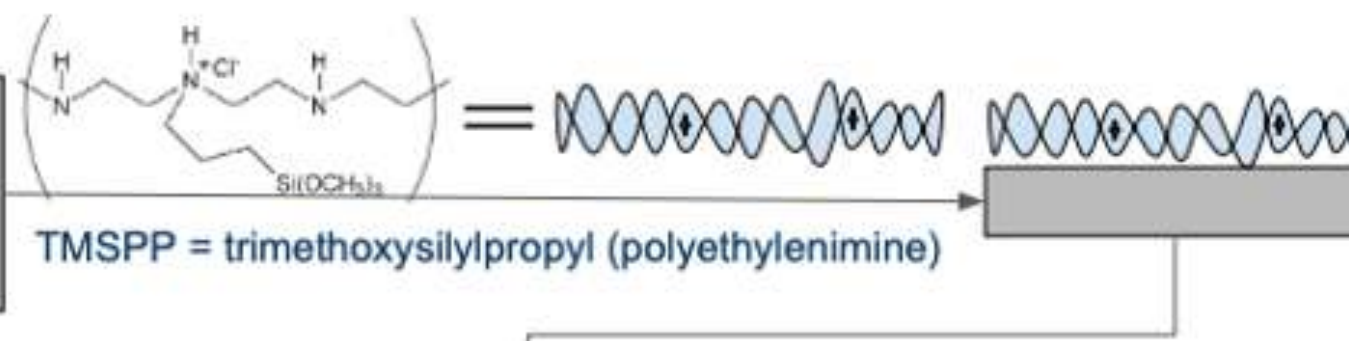

Allow ions to adsorb on surface for 8 hours in solutions with volumetric ratios of $1 \times 10^{-2} \mathrm{M} \mathrm{K}_{2} \mathrm{PdCl}_{4} \& 1 \times 10^{-2} \mathrm{M} \mathrm{HAuCl}_{4}$

(1.) (1.)

0000000000000000

$1: 0$

$1: 0.5$

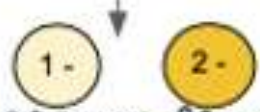

(1.)

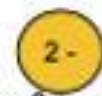

(2.)

(2.) 010000000000000000000000000000100000000000

\section{Reduce for 2 hours in $0.01 \%$ (w/v) sodium borohydride}

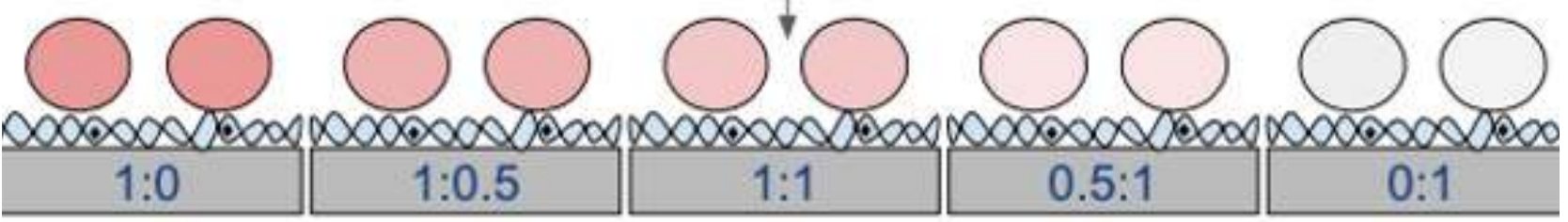




\section{Surface Morphology with AFM}
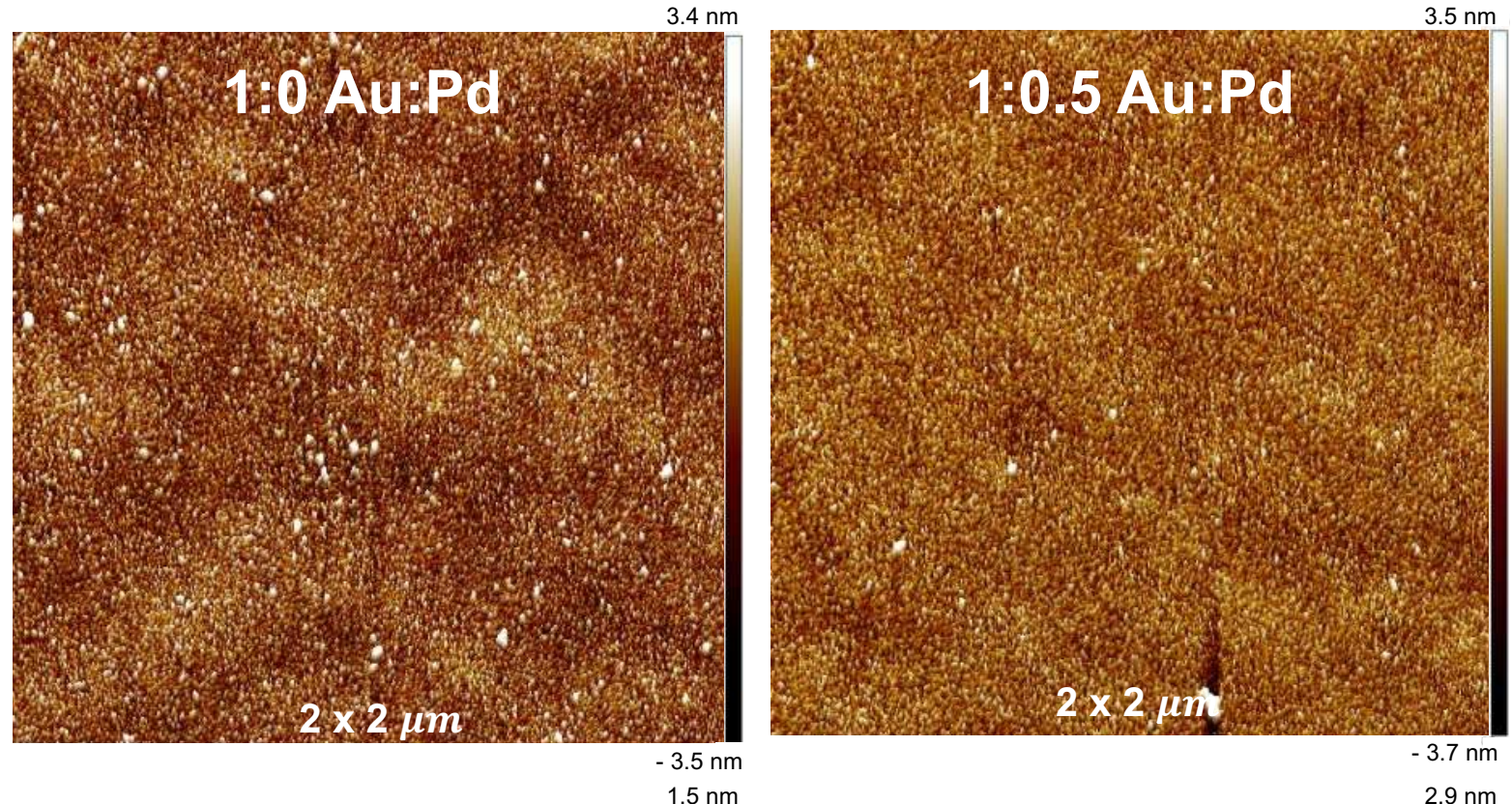

\begin{tabular}{|c|c|}
\hline Au:Pd Ratio & $\begin{array}{c}\text { Particle Size } \\
(\mathrm{nm})\end{array}$ \\
\hline $1: 0$ & 2.45 \\
\hline $1: 0.5$ & 2.99 \\
\hline $1: 1$ & 1.62 \\
\hline $0.5: 1$ & 2.63 \\
\hline $0: 1$ & 3.01 \\
\hline
\end{tabular}

1 1 1 A M P O
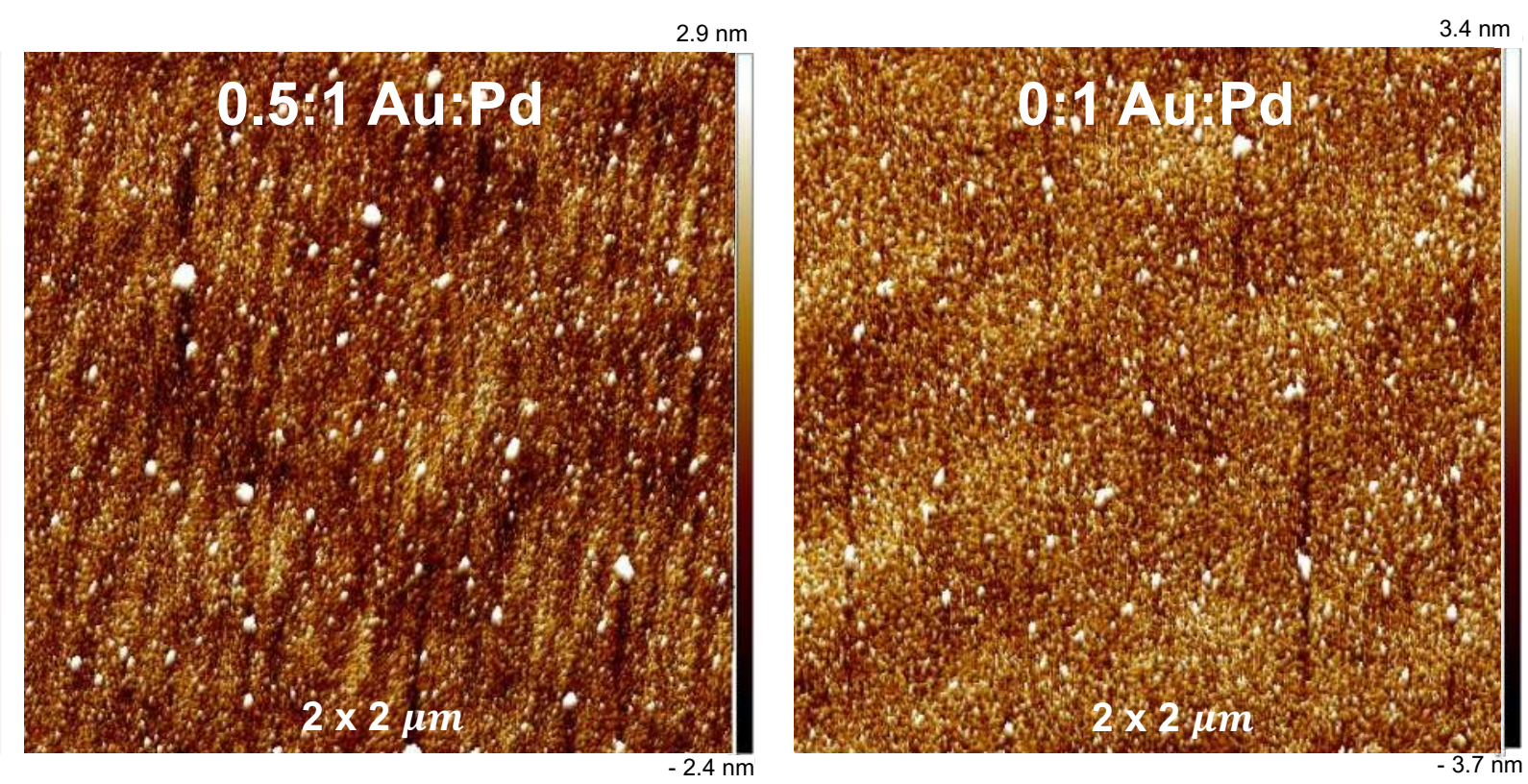

$2 \times 2 \mathrm{~mm}$ 


\section{Monitoring Surface Plasmons}

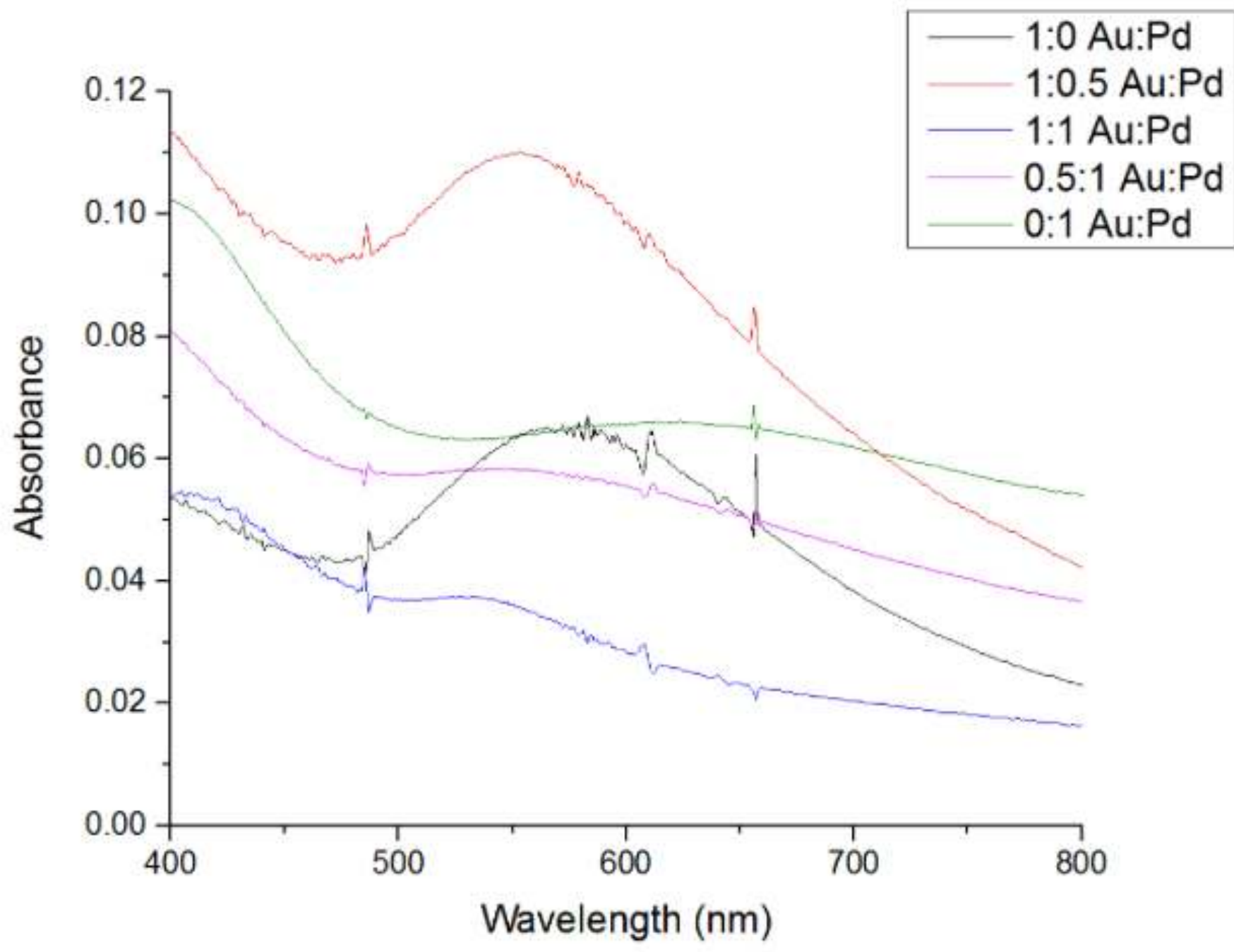

\begin{tabular}{|c|c|}
\hline $\begin{array}{c}\text { Au:Pd } \\
\text { Ratio }\end{array}$ & $\begin{array}{c}\text { Wavelength } \\
\text { Maximum (nm) }\end{array}$ \\
\hline $1: 0$ & 567 \\
\hline $1: 0.5$ & 540 \\
\hline $1: 1$ & 561 \\
\hline $0.5: 1$ & 598 \\
\hline $0: 1$ & 616 \\
\hline
\end{tabular}




\title{
Potential Nanoparticle Structure
}

\author{
Bimetallic NP Size > Au NP Size of $2.45 \mathrm{~nm}$
}

Bimetallic NP Wavelength < Au NP Wavelength of $567 \mathrm{~nm}$

1:0.5 Au:Pd

Size $=2.99 \mathrm{~nm}$

$\lambda=540 \mathrm{~nm}$

Core Shell Supported
1:1 Au:Pd

Size $=1.62 \mathrm{~nm}$

$\lambda=561 \mathrm{~nm}$

Core Shell Partially

Supported
0.5:1 Au:Pd

Size $=2.63 \mathrm{~nm}$

$\lambda=598 \mathrm{~nm}$

Core Shell Supported 


\section{Chemical Composition of NPs}

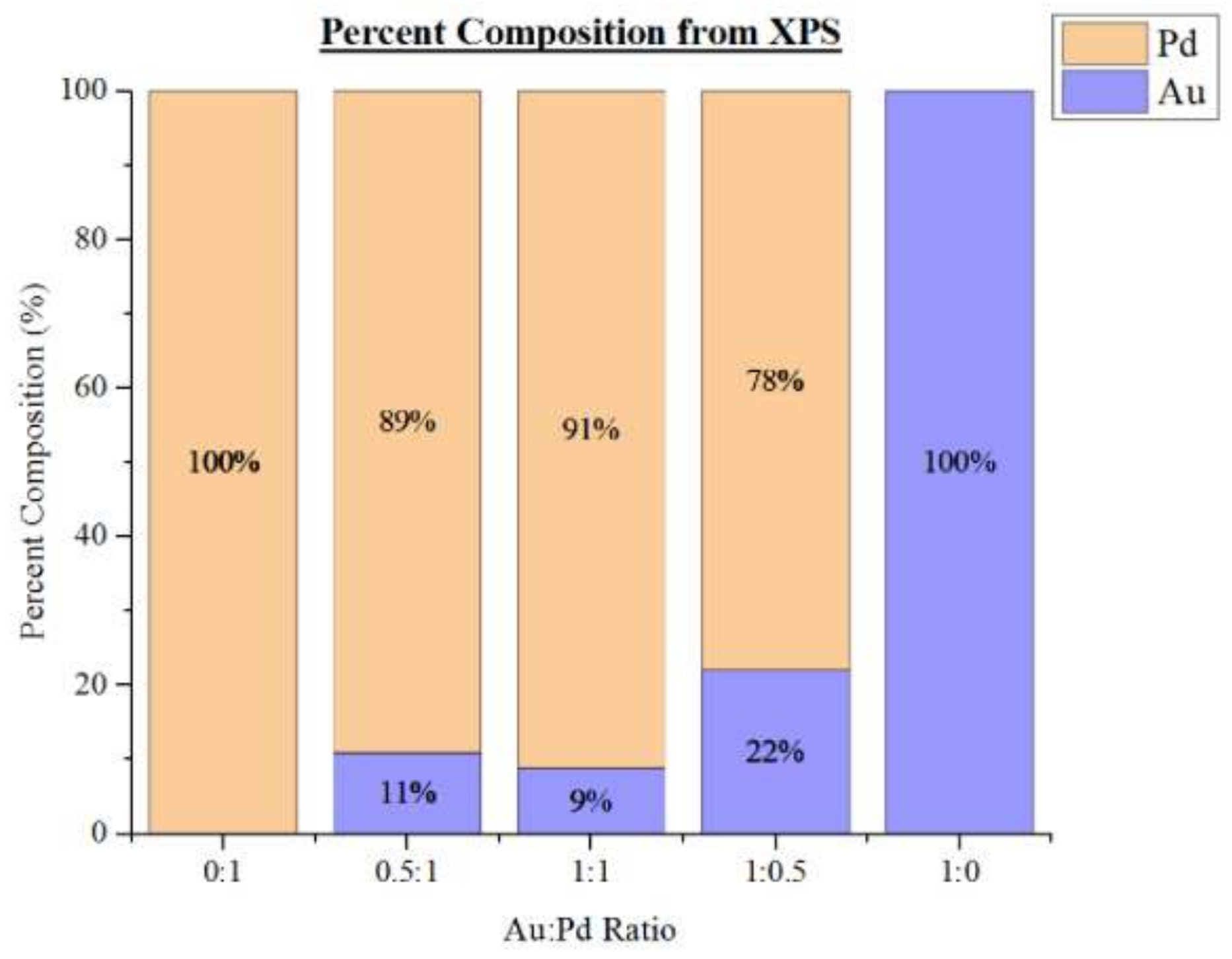




\section{Impedance response in presence of $\left[\mathrm{Fe}(\mathrm{CN})_{6}\right]^{3-/ 4-}$ as redox probe}
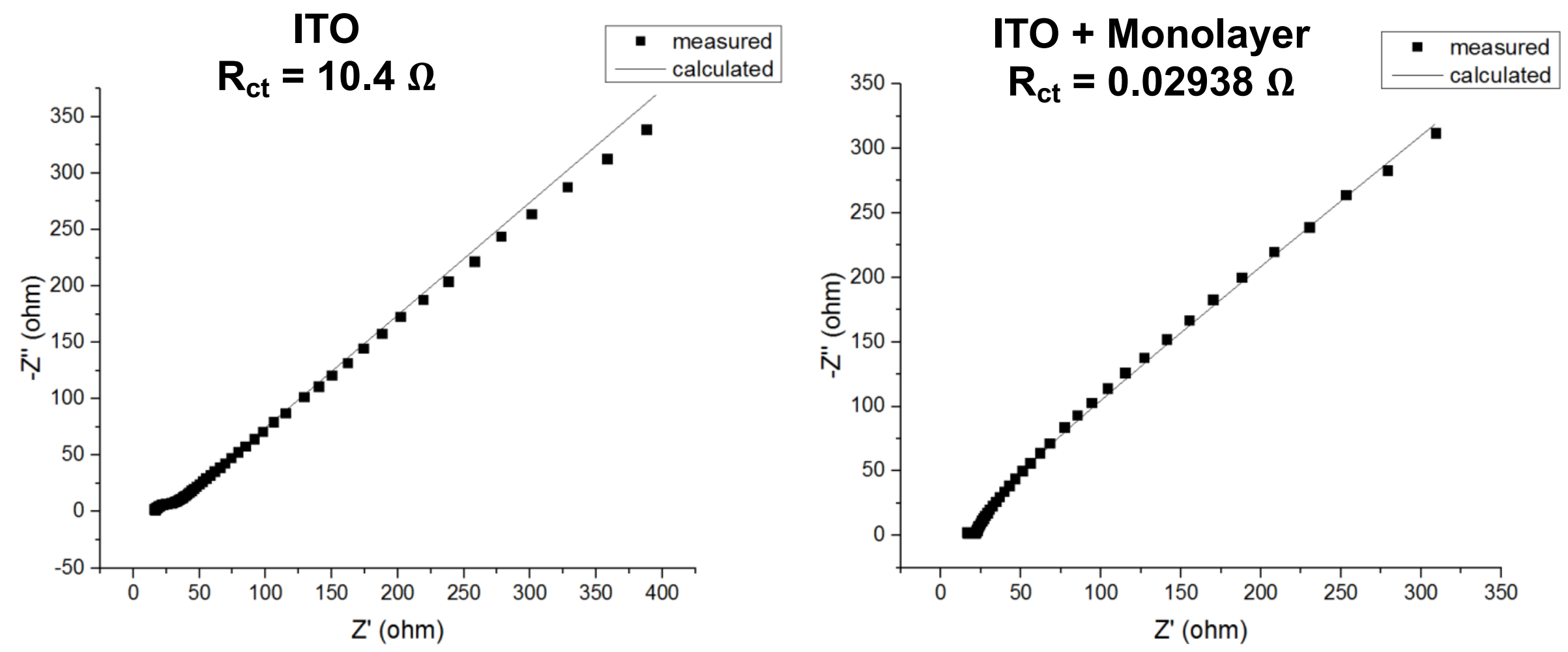


\section{Impedance response in presence of $\left[\mathrm{Fe}(\mathrm{CN})_{6}\right]^{3-/ 4-}$ as redox probe}

- $\equiv \quad\left[\mathrm{AuCl}_{4}\right]^{-}$
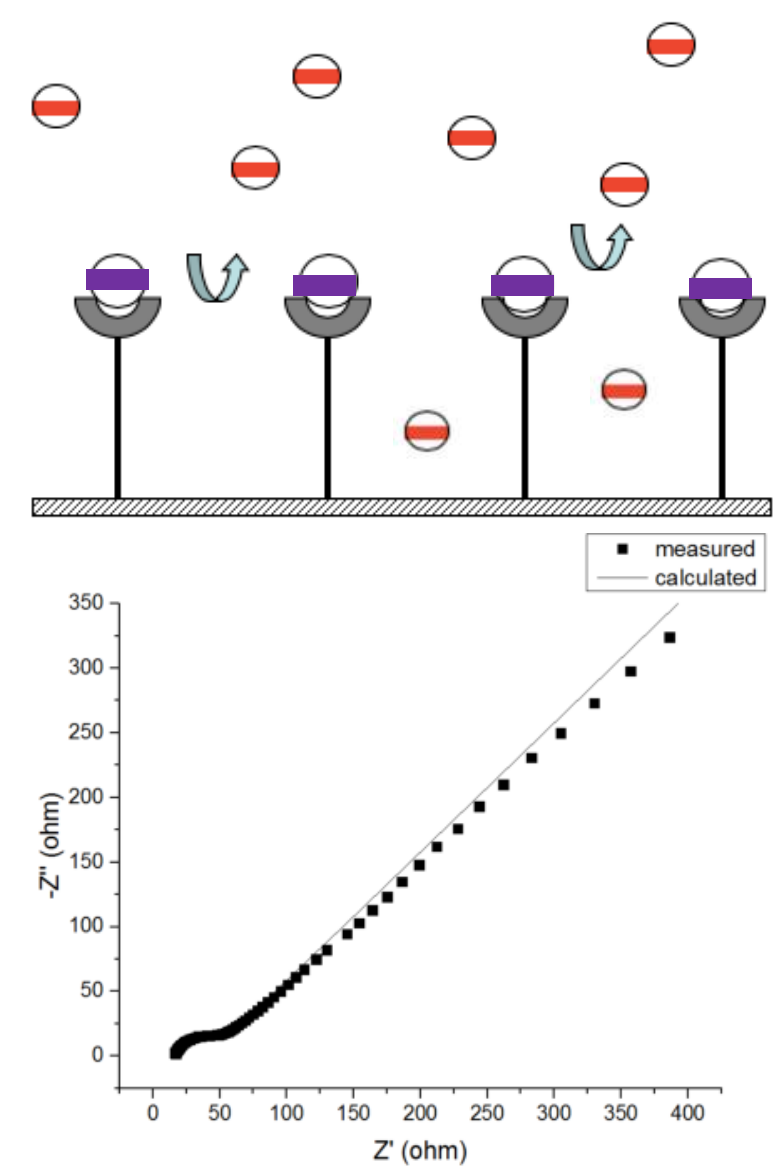

ITO + Monolayer + $\left[\mathrm{AuCl}_{4}\right]^{-}$ $R_{\mathrm{ct}}=26.3 \Omega$
$-\equiv\left[\mathrm{Fe}(\mathrm{CN})_{6}\right]^{3-4-}$

$=\quad \equiv \quad\left[\mathrm{PdCl}_{4}\right]^{2-}$
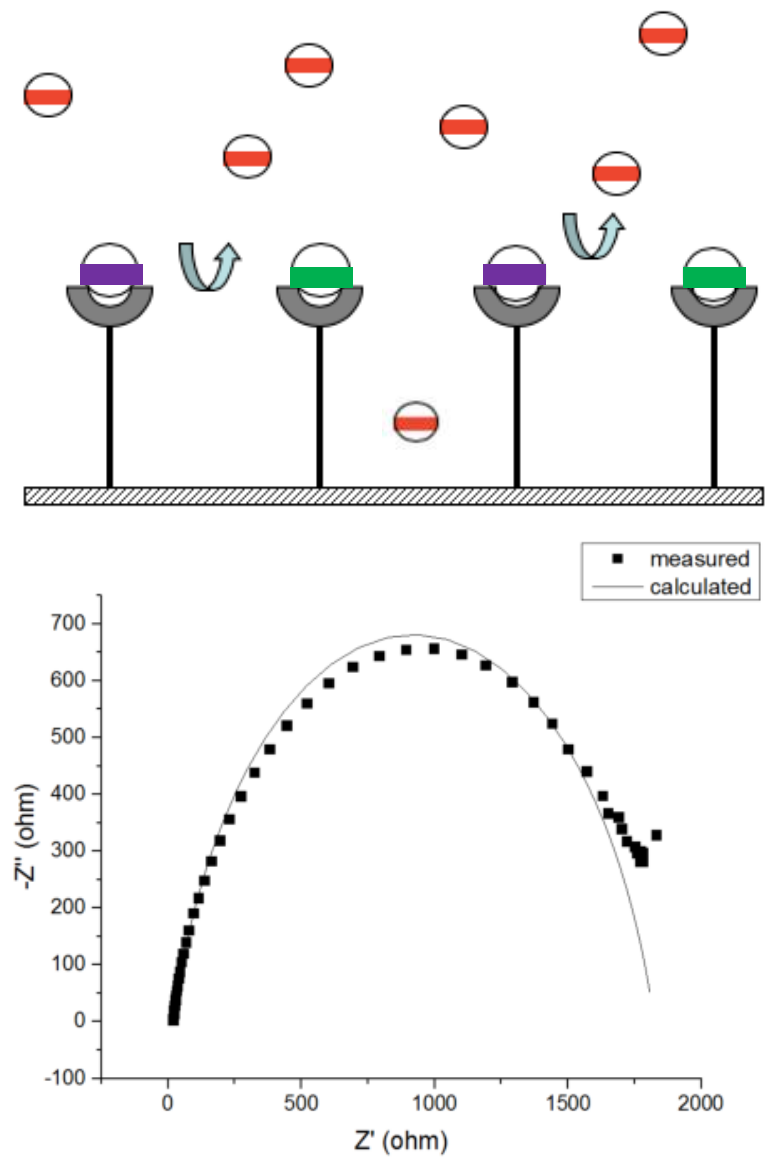

ITO + Monolayer + $\left[\mathrm{AuCl}_{4}\right]^{-}+\left[\mathrm{PdCl}_{4}\right]^{2-}$

$$
\mathbf{R}_{\mathrm{ct}}=1805 \Omega
$$
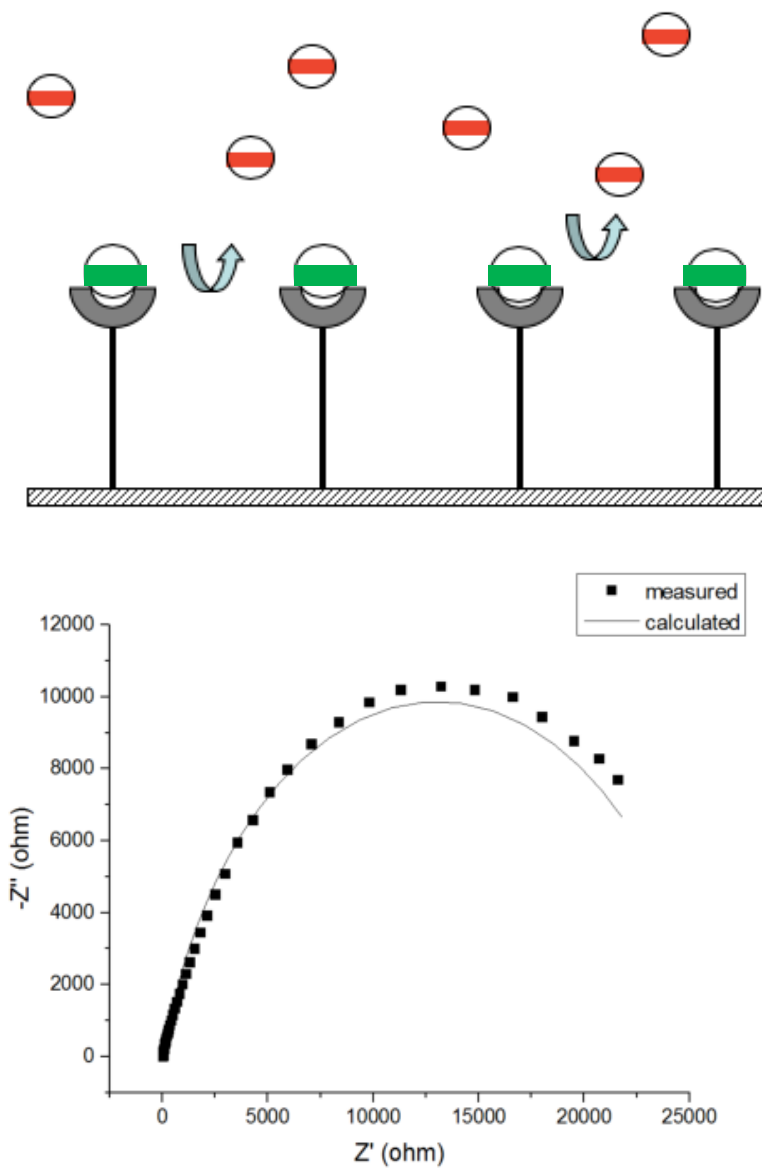

ITO + Monolayer $+\left[\mathrm{PdCl}_{4}\right]^{2-}$ $R_{\mathrm{ct}}=26050 \Omega$ 


\section{Analysis of Electrocatalytic Behavior}

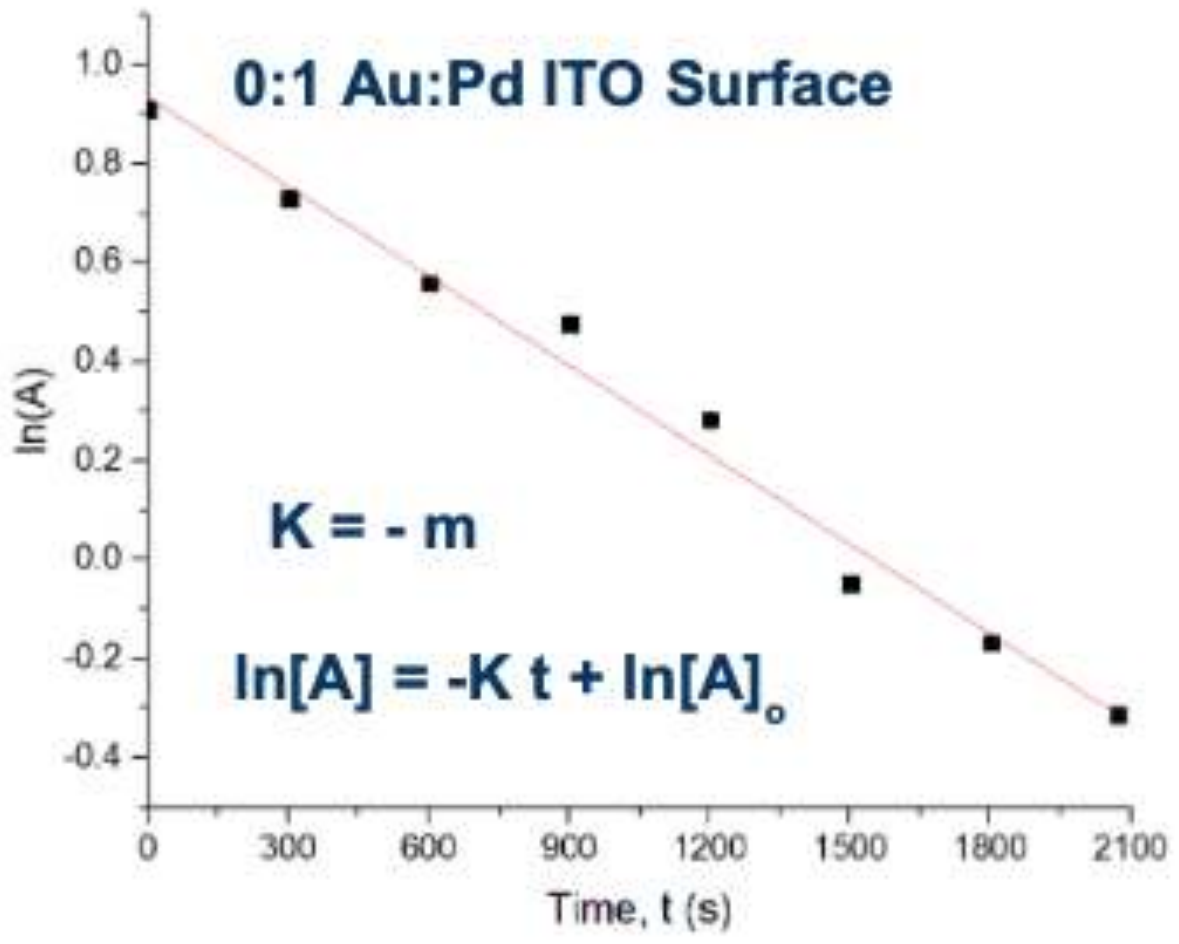

\begin{tabular}{|c|c|}
\hline Au:Pd Ratio & $\mathbf{K}_{\mathrm{app}} \times 10^{-4}(\mathbf{1} / \mathbf{s})$ \\
\hline $\mathbf{1 : 0}$ & 1.69 \\
\hline $\mathbf{1 : 0 . 5}$ & 3.17 \\
\hline $\mathbf{1 : 1}$ & 2.57 \\
\hline $\mathbf{0 . 5 : 1}$ & 2.28 \\
\hline $\mathbf{0 : 1}$ & 5.69 \\
\hline
\end{tabular}

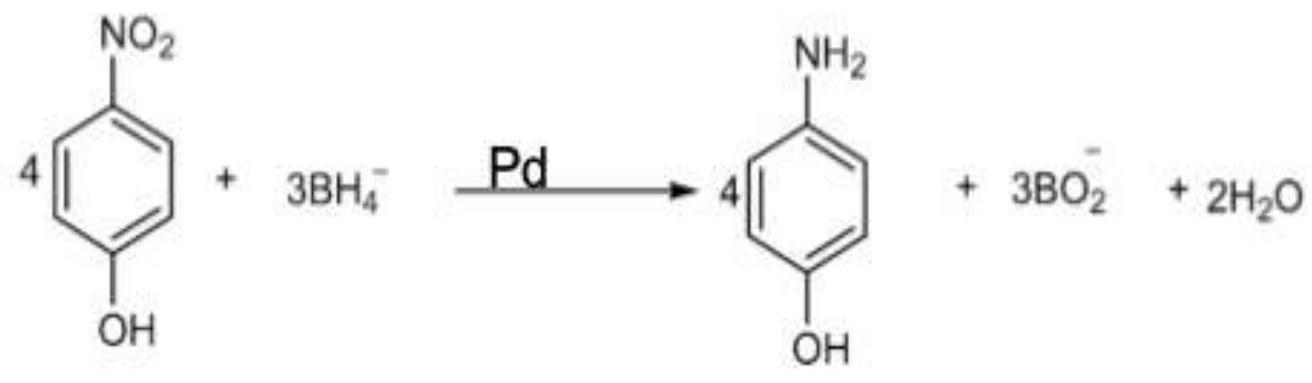

\section{Most Pd Exposure}

Moderate Pd Exposure

Least Pd Exposure 


\section{Analysis of Electrocatalytic Properties}
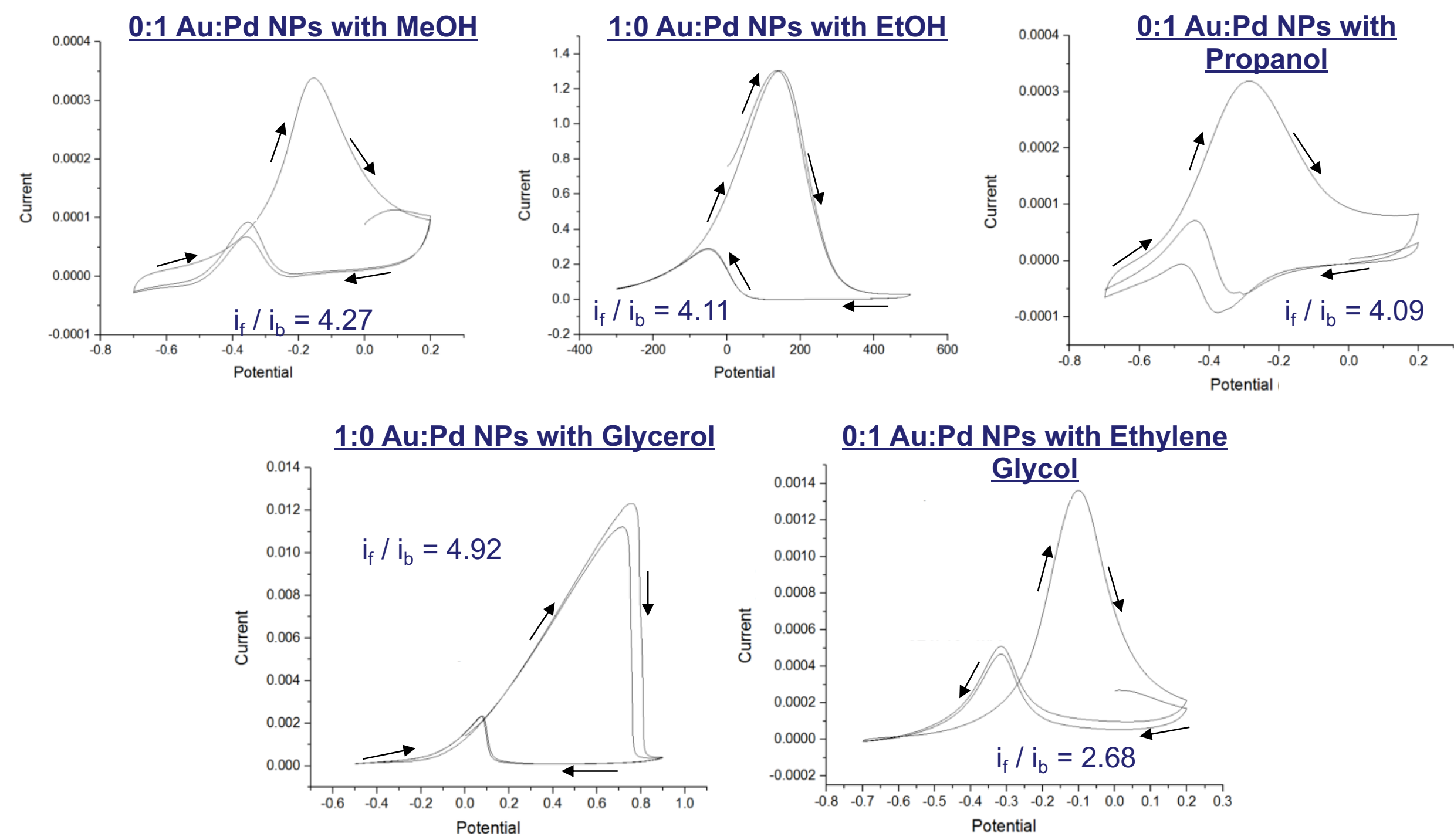


\section{Analysis of Electrocatalytic Properties}

\begin{tabular}{|c|c|c|c|c|c|c|}
\hline & Alcohol & Methanol & Ethanol & Propanol & $\begin{array}{c}\text { Ethylene } \\
\text { glycol }\end{array}$ & Glycerol \\
\hline & $1: 0$ & 4.07 & 4.11 & 3.95 & 1.15 & 4.92 \\
\cline { 2 - 7 } & $1: 0.5$ & 0.90 & 1.06 & 1.60 & 1.65 & 3.20 \\
\cline { 2 - 7 } Ratio & $1: 1$ & 1.42 & 1.01 & 2.04 & 1.03 & 1.90 \\
\cline { 2 - 7 } & $\mathbf{0 . 5 : 1}$ & 3.48 & 1.45 & 3.74 & 2.43 & 2.59 \\
\cline { 2 - 7 } & $\mathbf{0 : 1}$ & 4.27 & 1.10 & 4.09 & 2.68 & 2.71 \\
\hline
\end{tabular}




\section{Summary of Electrocatalytic Properties}

Current Ratio is

near the value for Pd NPs.

\section{Current Ratio is near the value for Au NPs.}




\section{Summary of Electrocatalytic Properties}

0.5:1 Au:Pd Ethylene glycol ratio was near the Pd ratio.
0.5:1 Au:Pd EtOH ratio was near the Pd ratio.

\section{$\frac{1: 1 \mathrm{Au}: \mathrm{Pd}}{\mathrm{EtOH} \text { ratio was }}$ near the $\mathrm{Pd}$ ratio.}

0.5:1 Au:Pd Glycerol ratio was near the Pd ratio.

1:1 Au:Pd Ethylene glycol ratio was near the Au ratio.

\section{More Pd Exposed}




\section{Summary}

1:0.5 Au:Pd

Size $=2.99 \mathrm{~nm}$

$\lambda=540 \mathrm{~nm}$

$\begin{array}{ll}\text { More Pd } & \text { K Value } \\ \text { Exposure } & \text { EtOH Ratio }\end{array}$

Well formed Pd shell with Au core

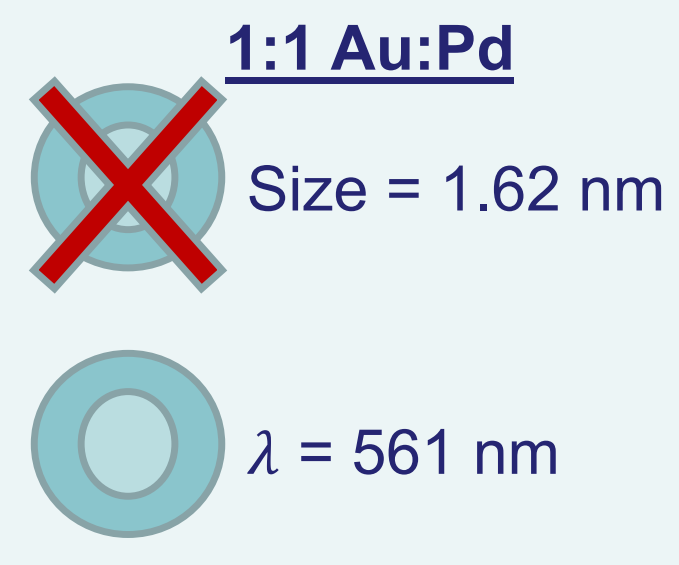

\begin{tabular}{c|l}
$\begin{array}{c}\text { More Pd } \\
\text { Exposure }\end{array}$ & EtOH Ratio \\
&
\end{tabular}

More Au Ethylene

Exposure glycol ratio

Poor Pd shell with Au core

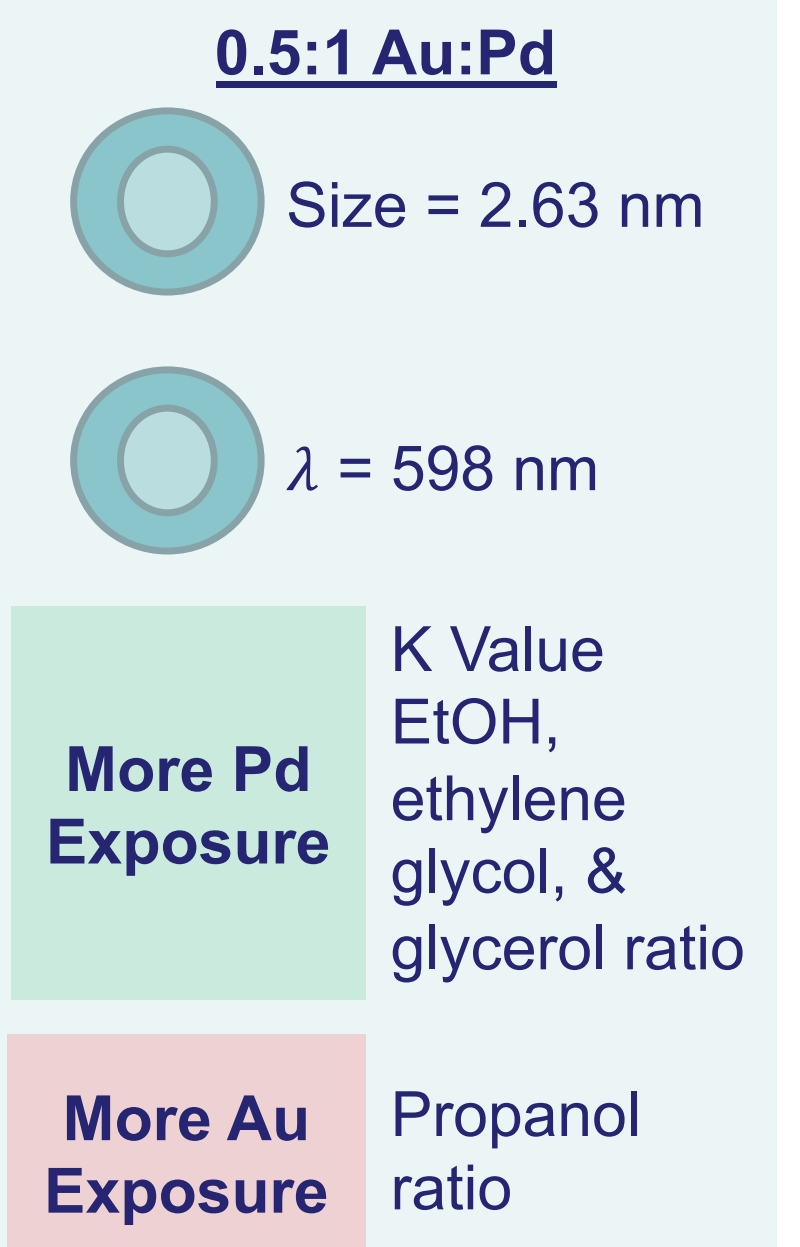

Moderate Pd shell with Au core 


\section{Au Nanorods Overview}

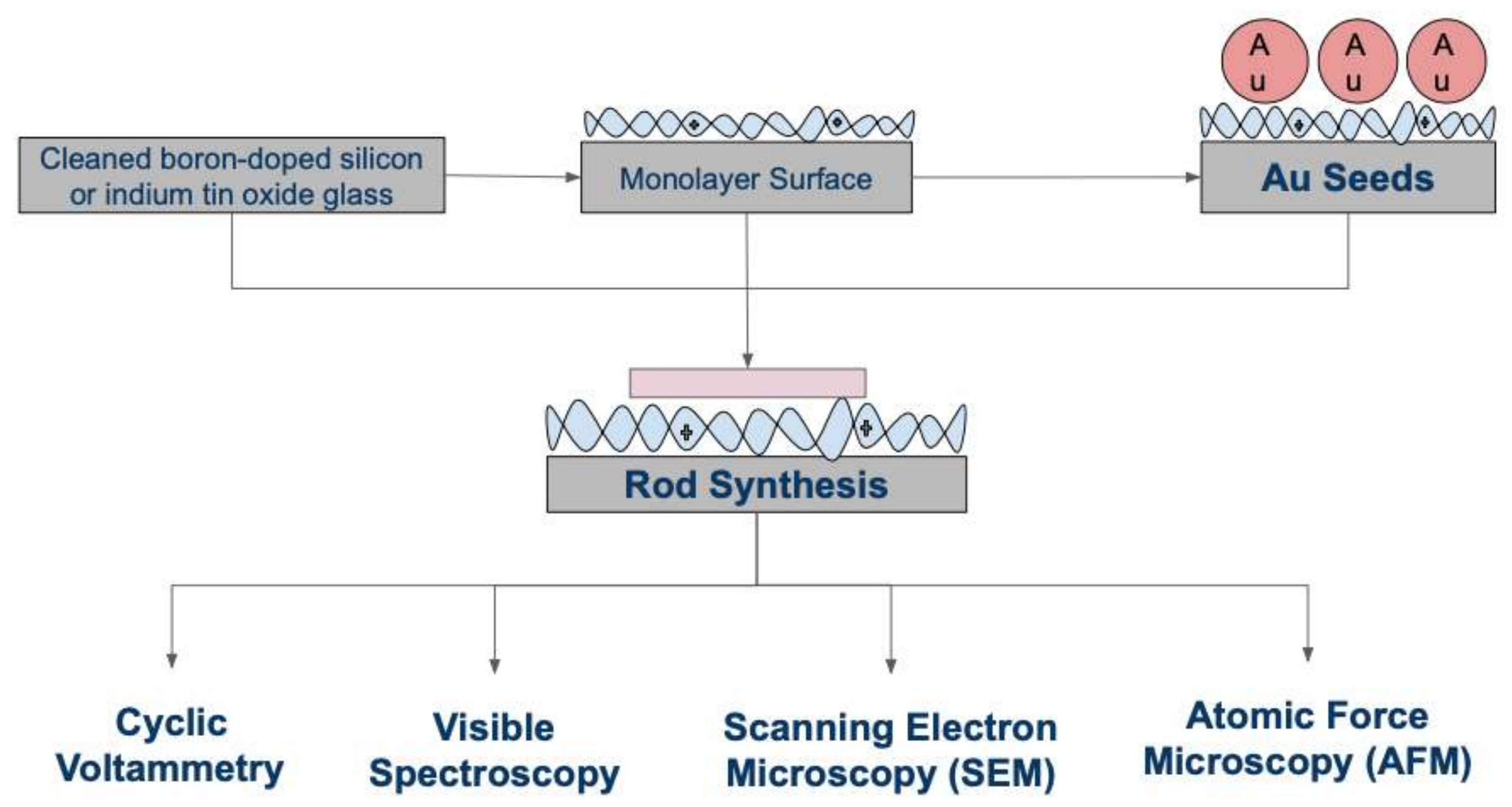




\section{Background}

SI/APTES surface

$\begin{array}{lllllll}\mathrm{NH}_{2} & \mathrm{NH}_{2} & \mathrm{NH}_{2} & \mathrm{NH}_{2} & \mathrm{NH}_{2} & \mathrm{NH}_{2} & \mathrm{NH}_{2}\end{array}$

\section{$\mathrm{CH}_{3} \mathrm{COOH}+\mathrm{DCC}$ \\ DMAP}

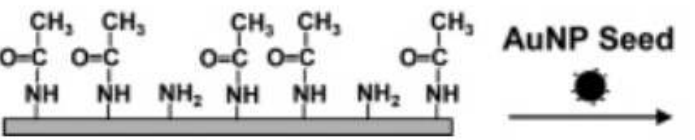

Mieszawska, A. J. AM. CHEM. SOC. 2006, 128, 5622-5623
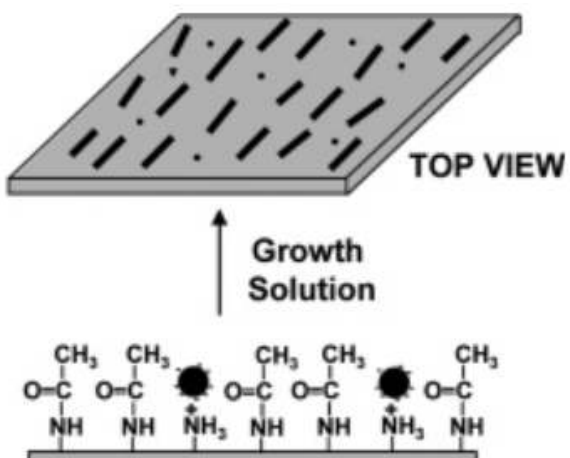

NH $\mathrm{NH}_{3} \mathrm{NH} \mathrm{NH} \mathrm{NH}_{3} \mathrm{NH}$
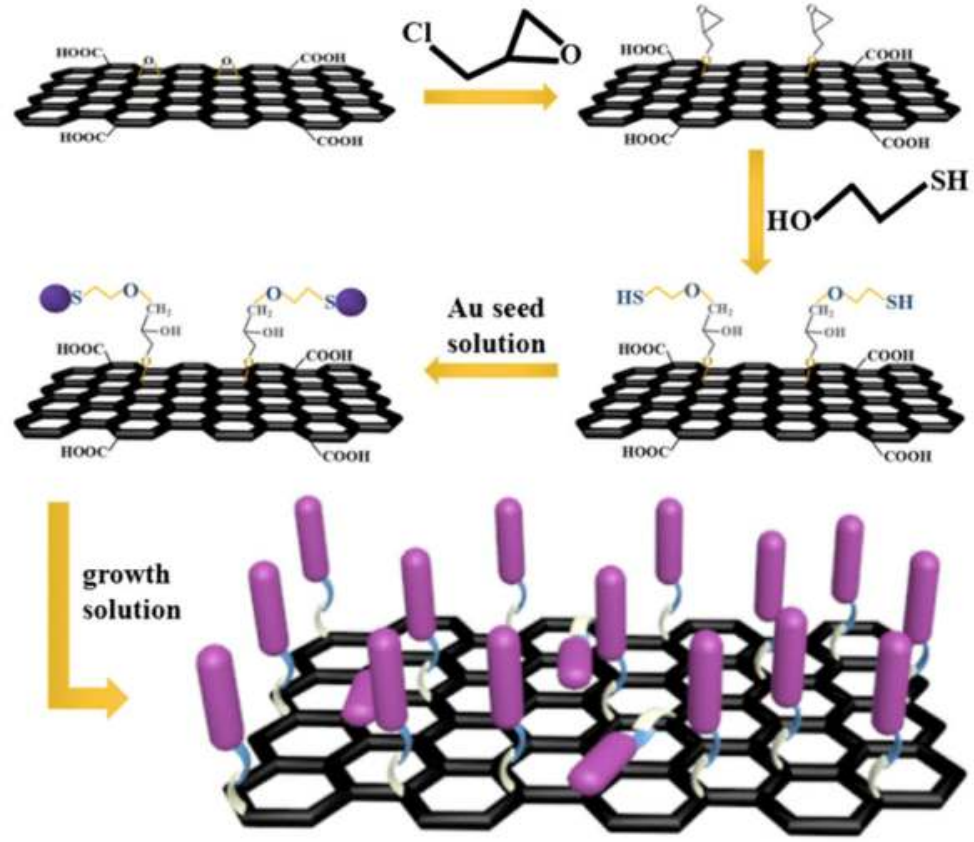

Guo, J. Appl. Organometal. Chem. 2019, 33, 4791

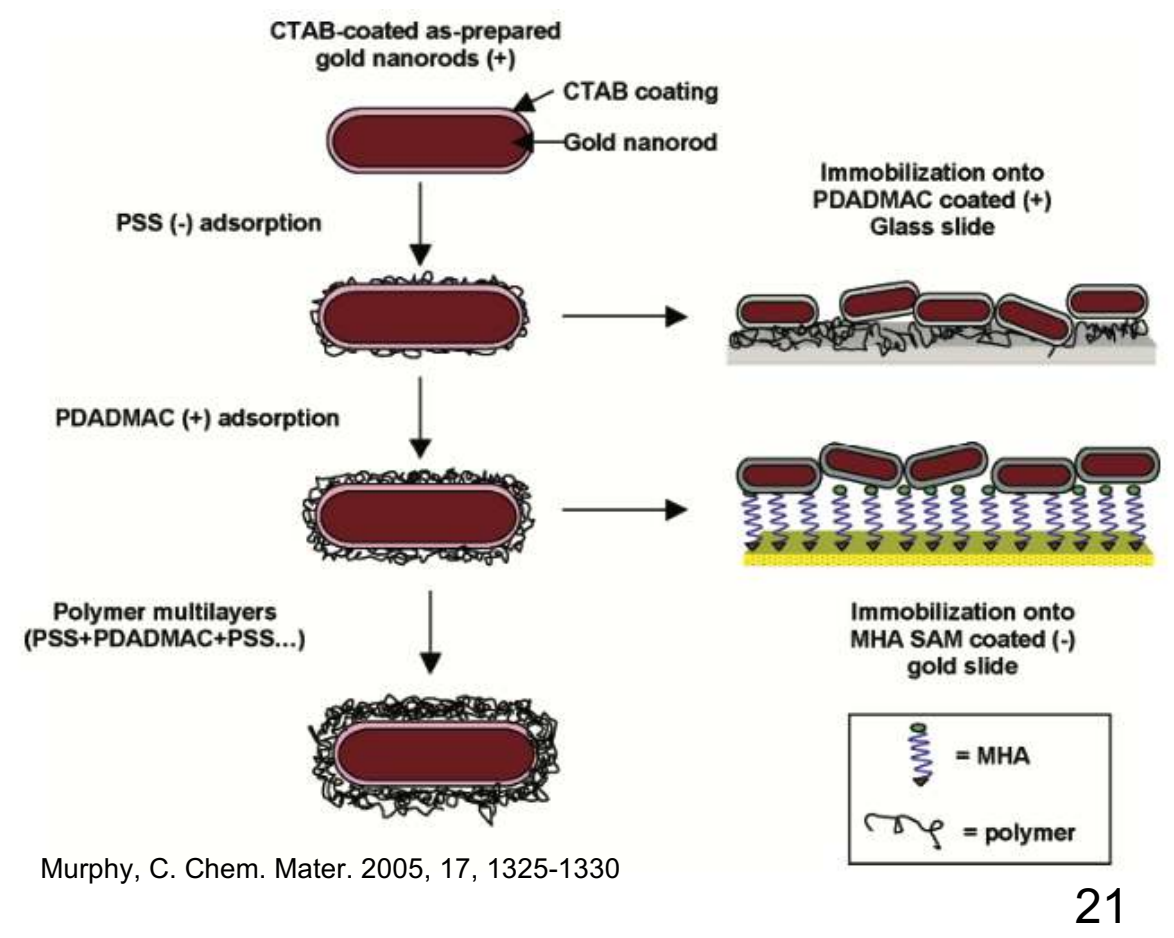




\section{Seed Synthesis}

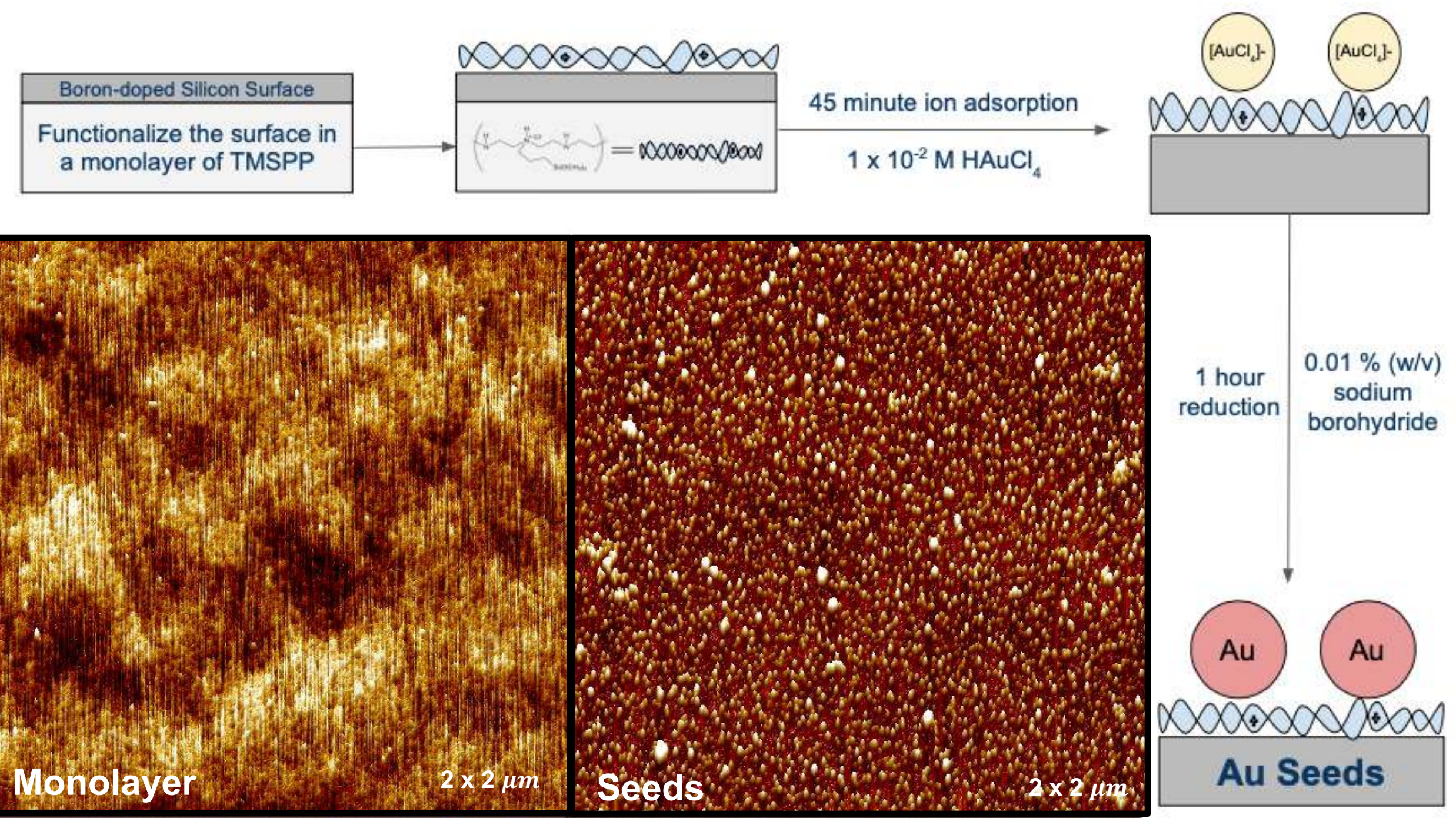

UnIVERSITY OF Michigan-DEARBorn 


\section{$\underline{\text { Rod Synthesis }}$}

Seed treatment

- Soak in $0.2 \mathrm{M}$ CTAB for $\sim 10$ minutes

Growth Solution A (GSA) from colloid paper

- $0.10 \mathrm{~g}$ of CTAB

- $5 \mathrm{~mL}$ of $1.5 \times 10^{-1} \mathrm{M}$ BDAC

- $0.20 \mathrm{~mL}$ of $4 \times 10^{-3} \mathrm{M} \mathrm{AgNO}_{3}$

- $5 \mathrm{~mL}$ of $1 \times 10^{-3} \mathrm{M} \mathrm{HAuCl}_{4}$

- $\quad 0.070 \mathrm{~mL}$ of $7.78 \times 10^{-2} \mathrm{M}$ Ascorbic Acid

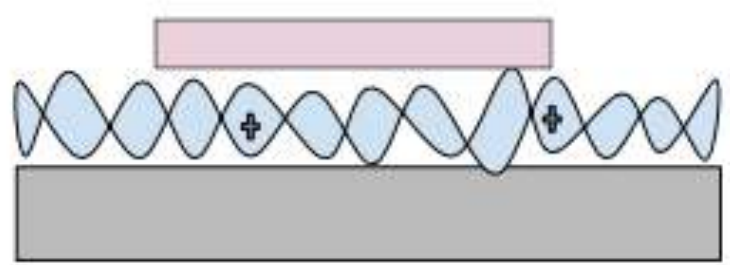

Cetyltrimethylammonium bromide (CTAB)

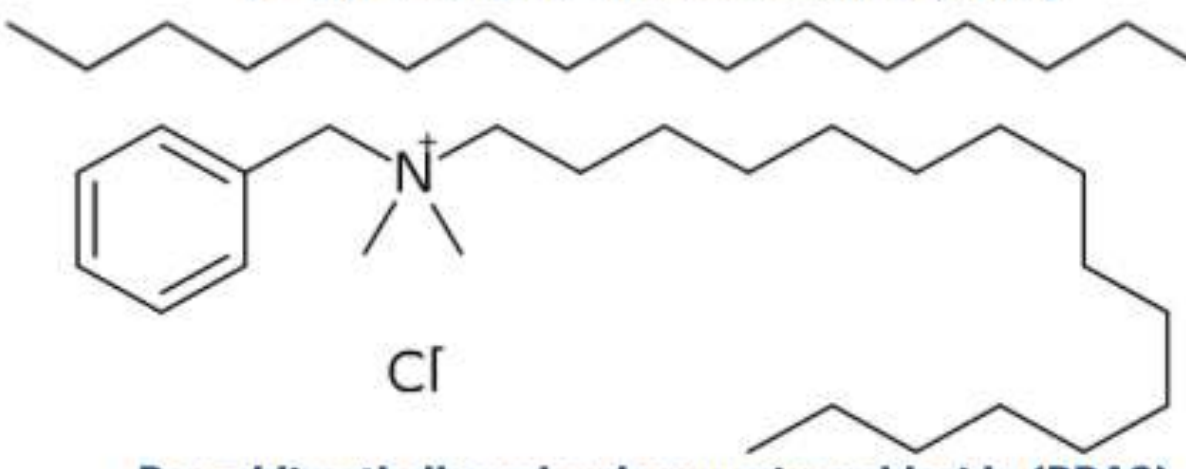

Benzyldimethylhexadecylammonium chloride (BDAC)

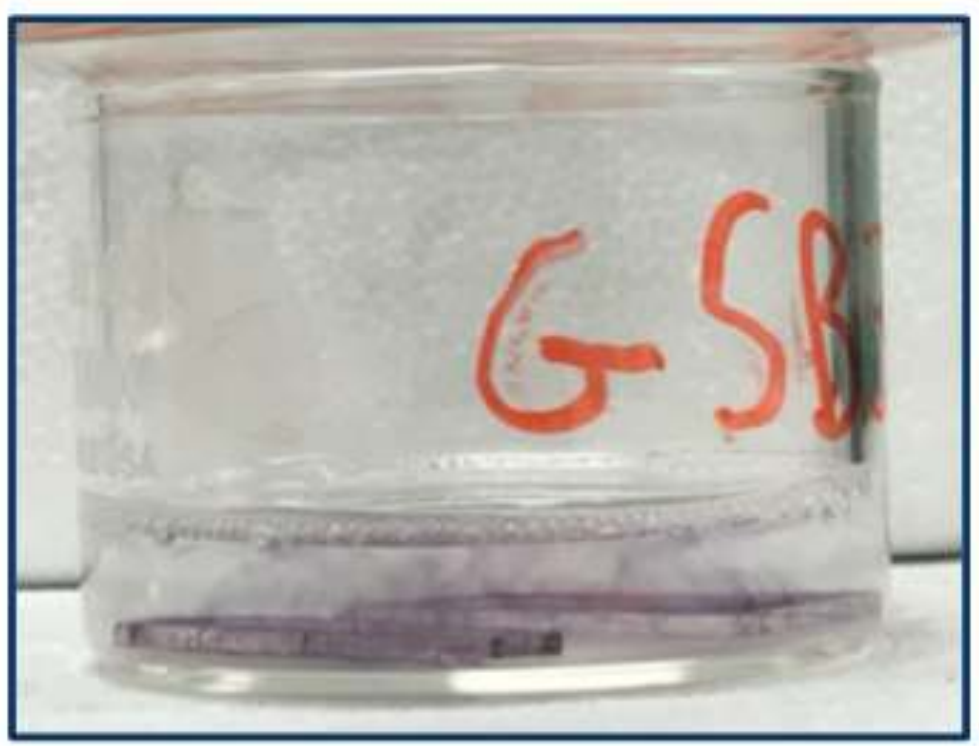

$\mathrm{Br}^{-}$ 


\section{Why surfactants?}
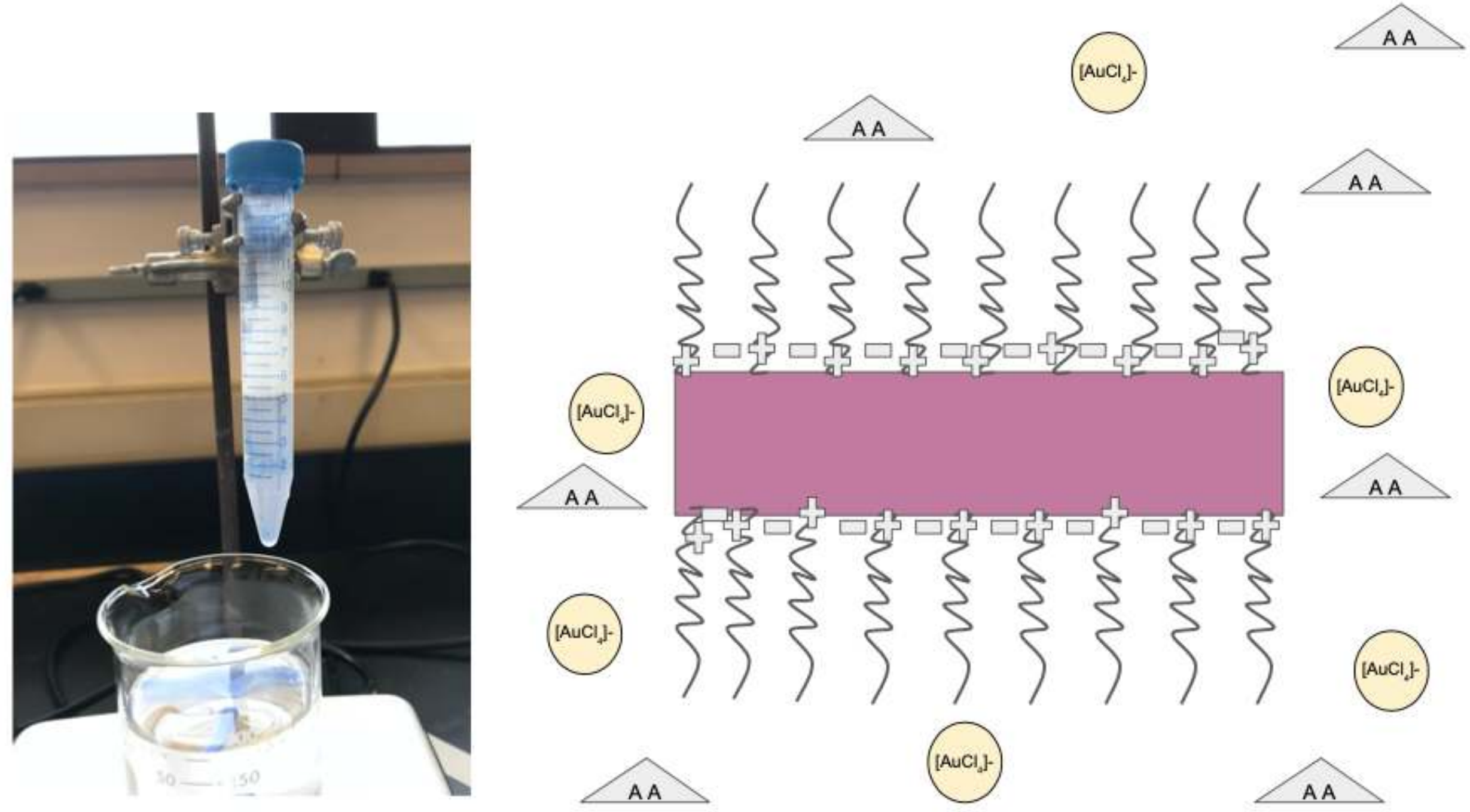


\section{Why silver?}

$\mathrm{Ag}+$

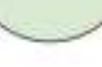

(ANCI, I.)

Mnso
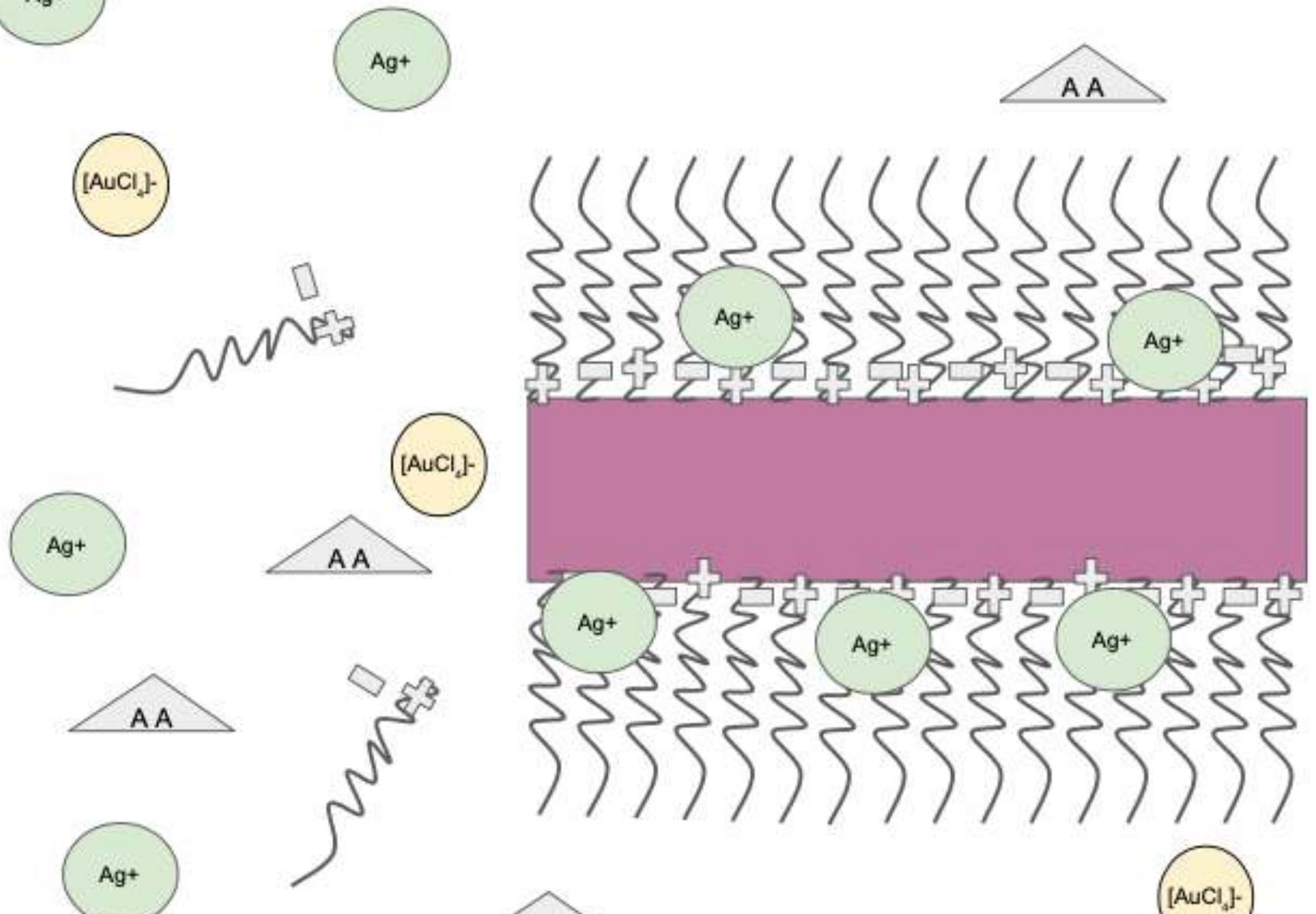
$-5 \leqslant$ Ag+
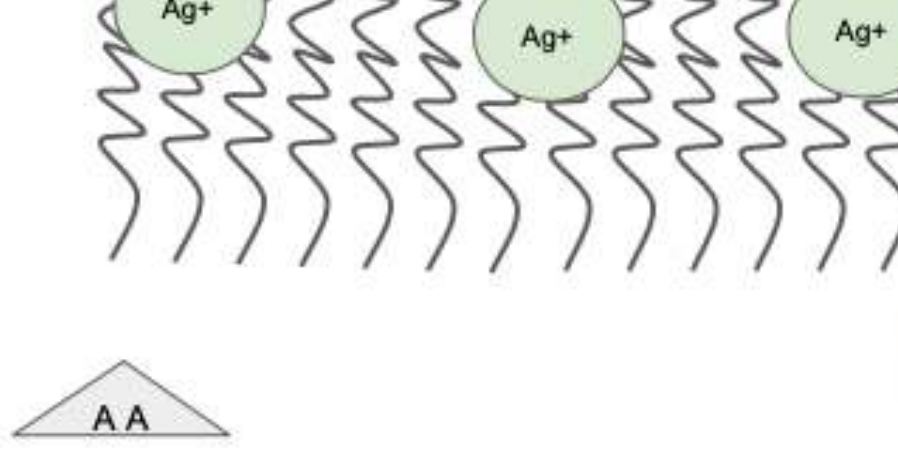

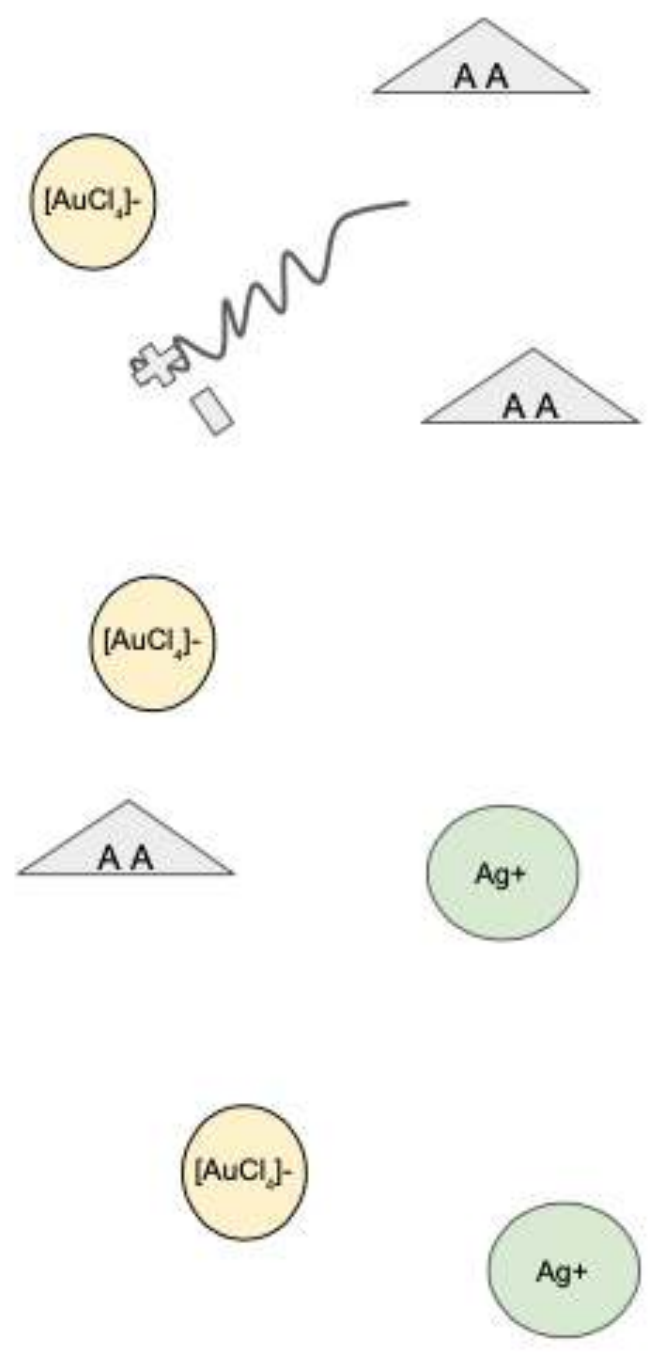




\section{Monitoring Surface Plasmons}
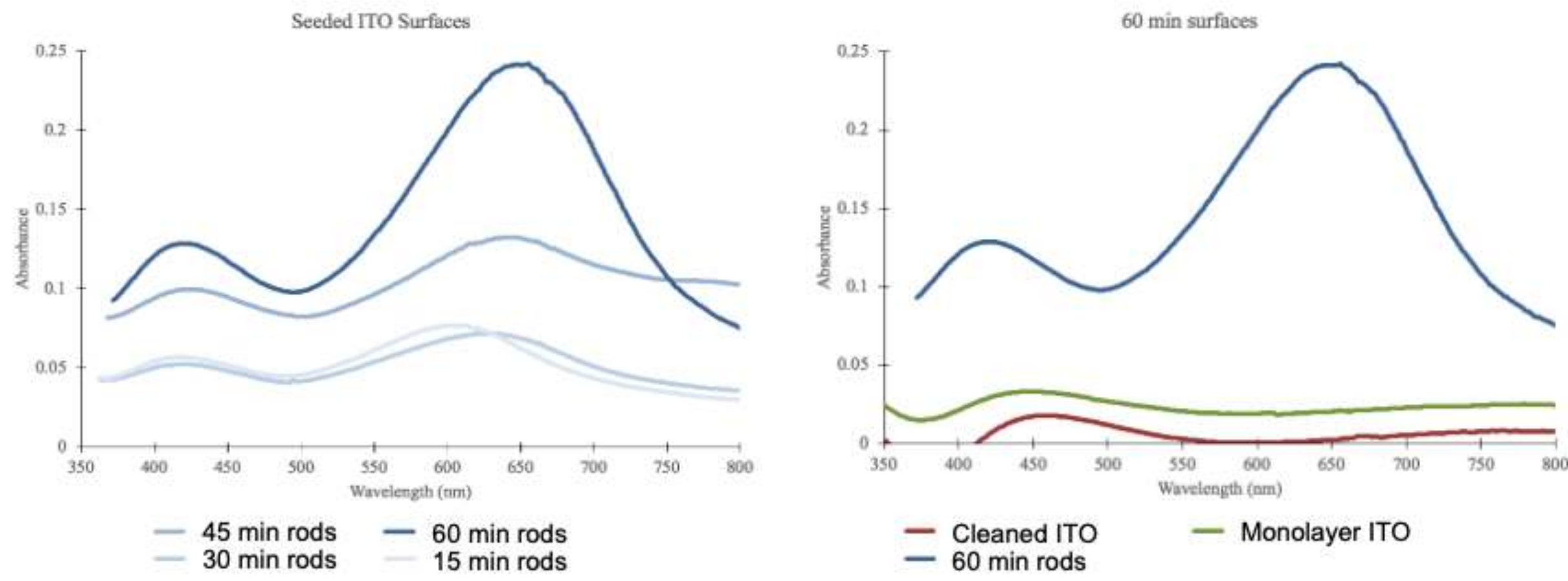

\begin{tabular}{|c|c|c|c|c|c|c|c|c|c|}
\hline \multirow{2}{*}{$\begin{array}{l}\text { Type of ITO } \\
\text { Time in GSA }\end{array}$} & \multicolumn{5}{|c|}{ Seeded ITO } & \multicolumn{2}{|c|}{ Monolayer ITO } & \multicolumn{2}{|c|}{ Cleaned ITO } \\
\hline & Seeds & $15 \mathrm{~min}$ & $30 \mathrm{~min}$ & $45 \mathrm{~min}$ & $60 \mathrm{~min}$ & $30 \mathrm{~min}$ & $60 \mathrm{~min}$ & $30 \mathrm{~min}$ & $60 \mathrm{~min}$ \\
\hline$\lambda_{\max } 1(\mathrm{~nm})$ & 417 & 420 & 421 & 409 & 414 & 456 & 486 & 449 & 450 \\
\hline$\lambda_{\max } 2(\mathrm{~nm})$ & - & 600 & 619 & 633 & 644 & - & - & - & - \\
\hline
\end{tabular}




\section{Nanorod Size with SEM}

\begin{tabular}{|c|c|c|c|c|c|}
\hline Type of ITO & \multicolumn{3}{|c|}{ Seeded ITO } & Monolayer ITO \\
\hline Time in GSA & $\begin{array}{c}\mathbf{1 5} \\
\mathbf{m i n}\end{array}$ & $\begin{array}{c}\mathbf{3 0} \\
\mathbf{m i n}\end{array}$ & $\begin{array}{c}\mathbf{4 5} \\
\mathbf{m i n}\end{array}$ & $\begin{array}{c}\mathbf{6 0} \\
\mathbf{m i n}\end{array}$ & $\mathbf{6 0} \mathbf{~ m i n}$ \\
\hline $\begin{array}{c}\text { Average Length, L } \\
(\boldsymbol{\mu} \mathbf{m})\end{array}$ & 34.8 & 46.5 & 104 & 61.1 & 61.9 \\
\hline $\begin{array}{c}\text { Average Width, W } \\
(\boldsymbol{\mu} \mathbf{m})\end{array}$ & 10.9 & 11.3 & 12.6 & 10.1 & 10.6 \\
\hline $\begin{array}{c}\text { Average Aspect } \\
\text { Ratio, L/W }\end{array}$ & 4.79 & 5.56 & 9.11 & 7.04 & 7.20 \\
\hline
\end{tabular}


Morphology with SEM
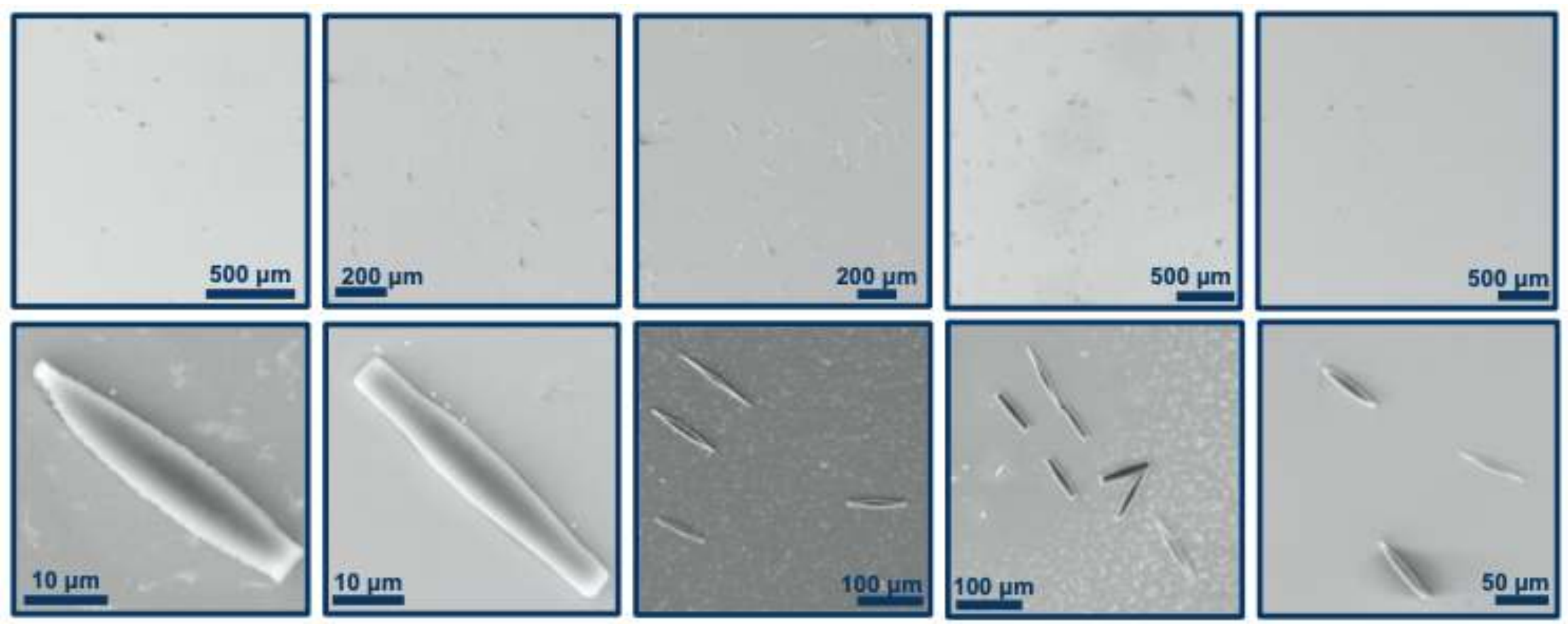

$15 \mathrm{~min}$

$30 \mathrm{~min}$

$45 \mathrm{~min}$

$60 \mathrm{~min}$

Monolayer 


\section{Analysis of Electrocatalytic Ability}

-5
-5
0




\section{Summary}




\section{Thank You!}

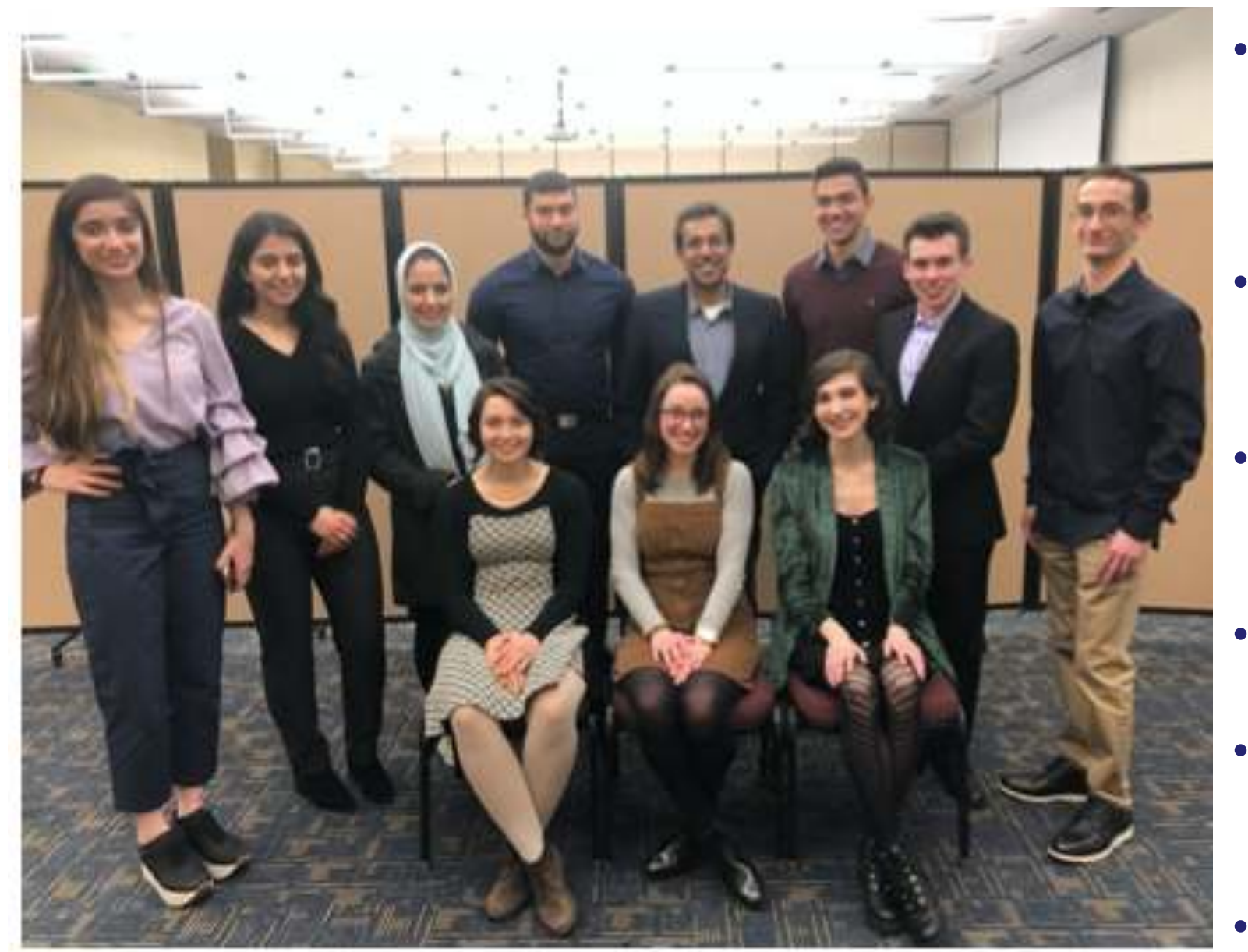

- Shout out to my subgroup: Diana Paicu, Aileen Peer, \& Ian Smith

- Rackham School of Graduate Studies, UM-Ann Arbor

- Office of Research \& Sponsored Programs, UMDearborn

- NSF-MRI (0619267, 0923023, \& 1039817)

- Office of the Vice President for Research, UM-Ann Arbor

- ACS-PRF Grant

UNIVERSITY OF MICHIGAN Office of the Vice President for Research
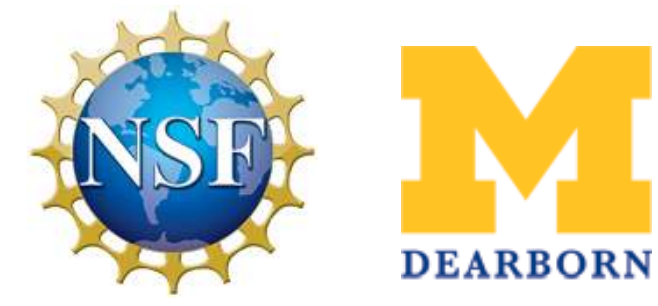


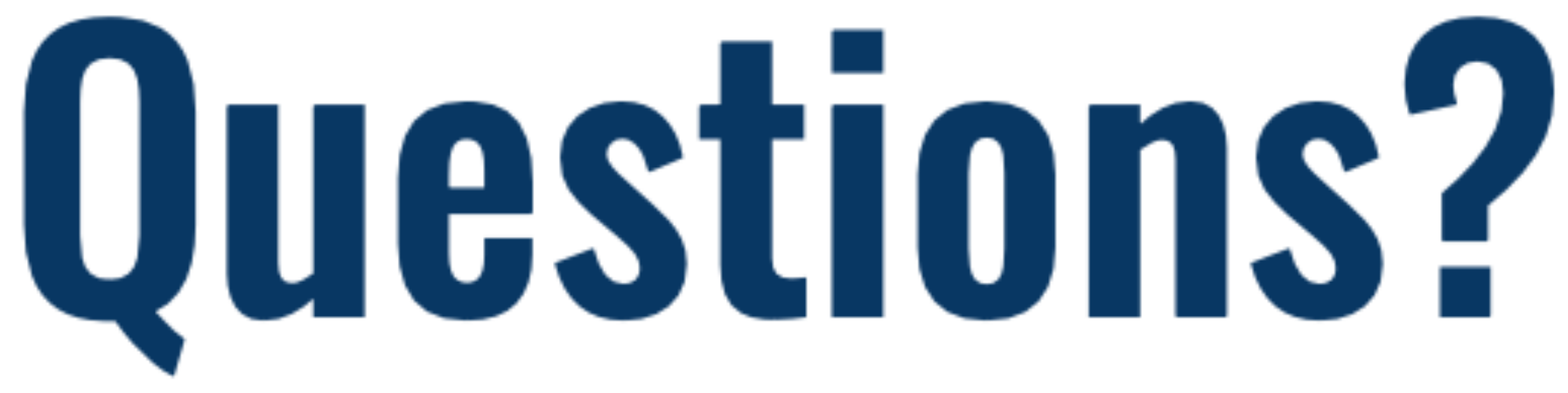




\section{Types of Nanoparticle Assemblies}

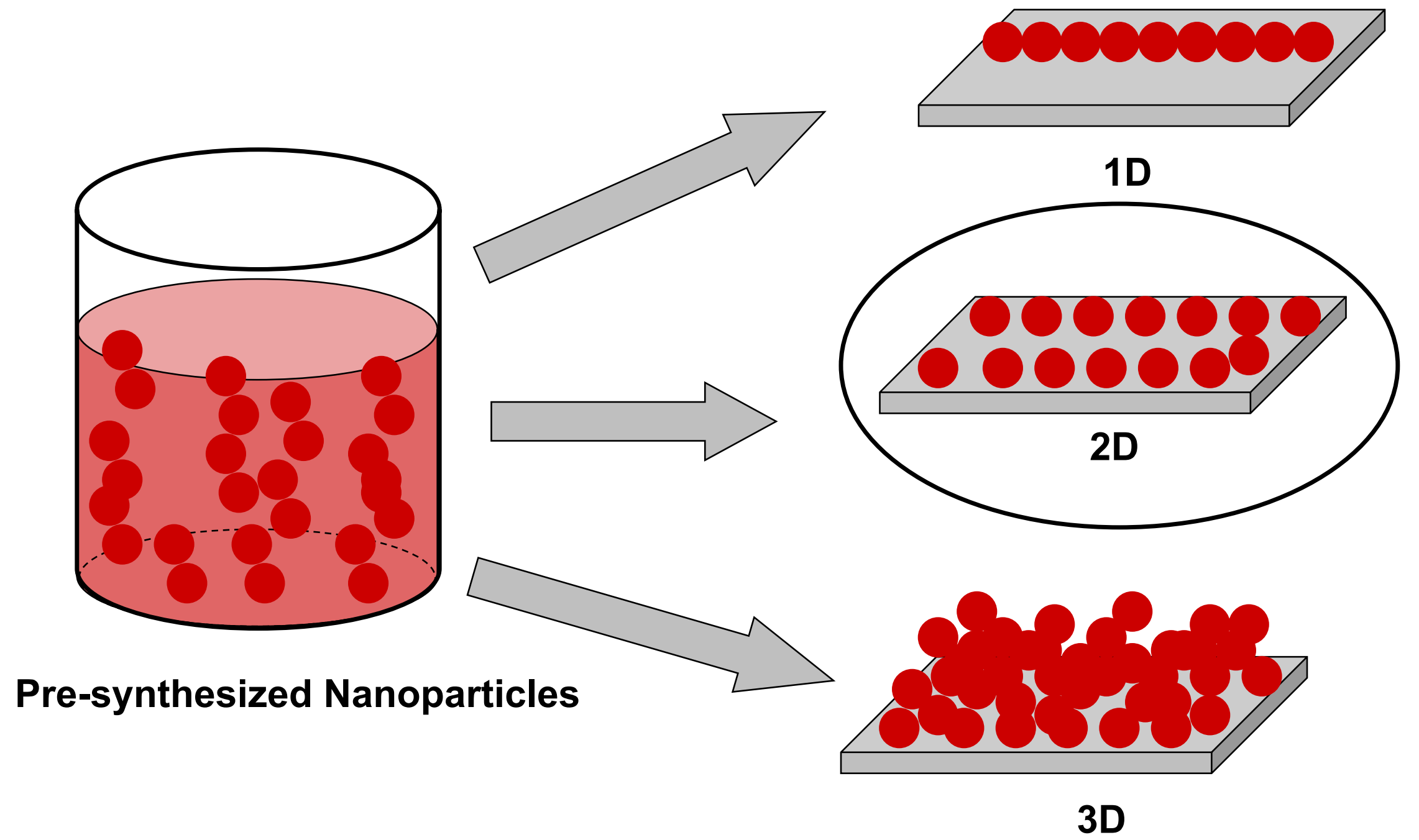




\section{Oxidation Pathways of Alcohols}

(a)

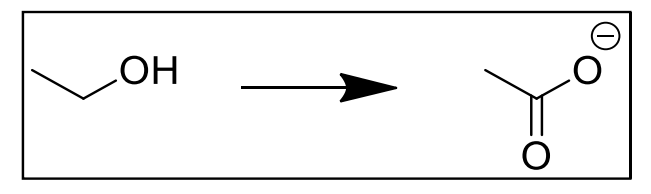

(b)

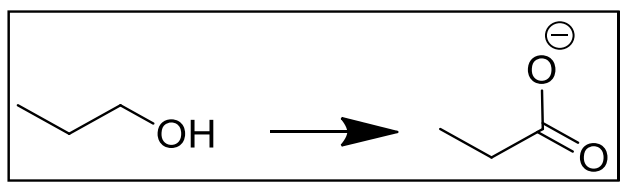

(c)

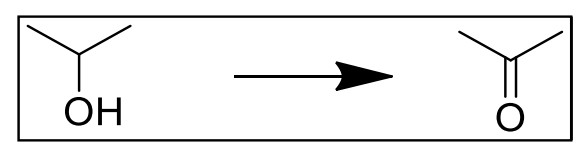

(d)

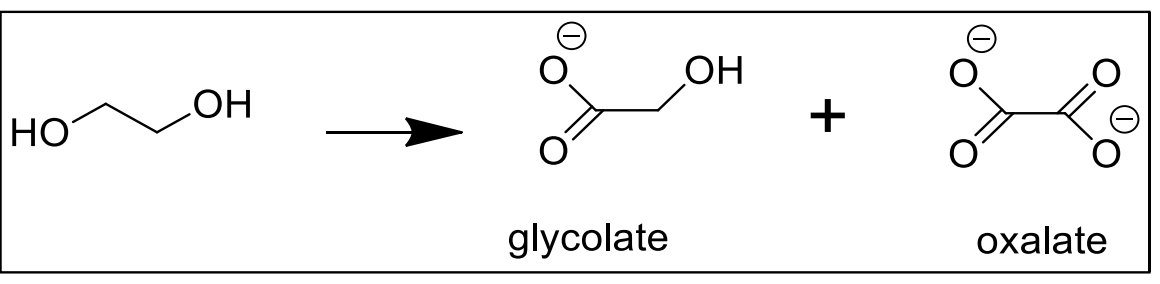

(e)

$\rightarrow$ glycerate

